\title{
The Adjoining Cell Mapping and Its Recursive Unraveling, Part II: Application to Selected Problems
}

\author{
R. S. GUTTALU and P. J. ZUFIRIA
}

\begin{abstract}
Several applications of the adjoining cell mapping technique are provided here by employing the adaptive mapping unraveling algorithm to analyze smooth and pathological autonomous dynamical systems. The performance of an implementation of recursive unraveling algorithm is also illustrated regarding its low memory requirements for computational purposes when compared with the simple cell mapping method. The applications considered here illustrate the effectiveness of the adjoining cell mapping technique in its ability to determine limit cycles and to unravel nonstandard dynamics. The advantages of this new technique of global analysis over the simple cell mapping method are discussed.
\end{abstract}

Key words: Nonlinear autonomous dynamical systems, simple cell mapping, limit cycles, adjoining cell mapping, adaptive integration, recursive algorithms.

\section{Introduction}

This paper is a sequel to [11] in which theoretical aspects of the concept of adjoining mapping in the cellular state space (see [4]) have been developed for nonlinear autonomous differential equations of the form

$$
\dot{\mathbf{x}}=\mathbf{F}(\mathbf{x}), \quad \mathbf{x} \in \mathbf{R}^{N}, \quad \mathbf{F}: \mathbf{R}^{N} \rightarrow \mathbf{R}^{N}, \quad \mathbf{F} \in C^{0},
$$

where $\mathbf{x}$ is an $N$-dimensional state vector and the vector field $\mathbf{F}$ is, in general, a nonlinear function of $\mathbf{x}$. The primary objective here is to complement this theory with applications which analyze the performance of the method of adjoining cell mapping and its adaptive and recursive unraveling implementations when applied to (1). An adjoining cell mapping associated with (1) may be described by

$$
\mathbf{z}(n+1)=\mathbf{C}_{A}(\mathbf{z}(n)), \quad \mathbf{C}_{A}: S \rightarrow S, \quad n \in \mathbf{Z},
$$

where $\mathbf{z}$ is an $N$-dimensional cell state vector in the cellular state space $S$ and $\mathbf{C}_{A}$ is a mapping from a set of integers to a set of integers. The mapping $\mathbf{C}_{A}$ is such that a cell in $S$ maps to an adjoining cell. Several classes of applications of the map $\mathbf{C}_{A}$ are considered in this paper to show its effectiveness in capturing the essential dynamics of the original system. We begin with the application of the adjoining mapping technique in Section 2 to smooth autonomous dynamical systems to illustrate and to interpret the results of practical implementation of the algorithm provided in Section 3.2 (adaptive mapping unraveling algorithm) and Section 4.3 (recursive 
unraveling algorithm) of Zufiria and Guttalu [11]. Two- and three-dimensional systems possessing multiple limit cycles are considered including the classical van der Pol equation. In all these examples, the method of adjoining mapping will be shown to replace each limit cycle by a single group of connected periodic cells. This is a clear advantage over the simple cell mapping method which usually replaces a limit cycle by several groups of disconnected periodic cells. For the particular discretization of state space chosen for these examples, our method also locates unstable limit cycles.

The method of adjoining mapping is applied next to study a simple Hamiltonian system in Section 2.5. It is well-known that time-discretization of such systems are not necessarily Hamiltonian, see [6]. With a further discretization of state space for the purpose of cell mapping analysis, some original properties such as the existence of nonisolated periodic solutions will lose their meaning. The results of the simple cell mapping method are particularly difficult to interpret since each closed orbit may be replaced by a number of groups of periodic cells depending again on the integration time $T_{S}$ chosen for obtaining the mapping from (1). The technique of adjoining mapping seems to provide a 'cleaner' set of results since it replaces a closed orbit in the cellular space by a single connected group of periodic cells. As a final example of smooth dynamical system, in Section 2.6 we consider the Lorenz equation when it possesses a chaotic attractor. Simple cell mapping results for both Hamiltonian and chaotic systems usually provide a large number of periodic solutions. Since this may also be the case even when regular dynamics are analyzed depending on the duration of integration, the simple cell mapping method may not yield information needed to distinguish among these cases. It has already been pointed out that the adjoining cell mapping method provides a very neat description of regular dynamics. For both Hamiltonian and chaotic systems, the adjoining mapping method provides a number of periodic groups adjoining to one another (unlike in the case when limit cycles are present). This feature may indicate the presence of 'complicated' dynamics or dynamics very sensitive to state space discretization.

Another class of examples considered in Section 3 is the type of dynamical systems studied in references. $[2,8,9,10]$ in connection with the problem of determining all the zeros of a nonlinear vector function. Such systems (referred to as nonstandard or pathological dynamical systems) possess 'fast' dynamics and exhibit pathological characteristics usually not found in commonly encountered dynamical systems. Because of the rapidly changing nature of its vector field (which may be unbounded on singular manifolds of the system), the determination of system global dynamics is very challenging and poses severe numerical difficulties while tracking down system trajectories. No matter how carefully the integration of system equations is carried out, the method of simple cell mapping applied to such a system may generate a domain of attraction in the cell state space which contains disconnected subsets that do not correspond very precisely with the original domain of attraction associated with an invariant set of the dynamical system, see [8, 10]. It will be shown here that the method of adjoining mapping is very well suited for studying the global behavior of this class of dynamical systems, especially in providing meaningful domains of attraction. In addition, the method also reveals some details in the system's global behavior which have been theoretically predicted.

Comparative properties of the simple cell mapping and the adjoining cell mapping methods are considered in Section 4. Overall, the new method of cell mapping analysis has been found to provide promising results for describing both regular and nonstandard dynamics in cellular state space. However, its strength lies in its ability to analyze nonstandard dynamics. Concluding remarks of this paper appear in Section 5. 


\section{Application Involving Smooth Dynamics}

For the examples considered in this paper, an adjoining cell mapping for the given dynamical system is computed by employing a fourth-order Runge-Kutta method and the integration time is obtained by the cell-time finding algorithm presented in Section 3.2 of [11].

In this section, we consider autonomous dynamical systems whose vector field evolves smoothly in state space. Our main objective here is to apply the computational adjoining mapping algorithm to locate limit cycles in the cellular state space and to interpret results for Hamiltonian and chaotic systems.

\subsection{Example 1. Limit Cycle}

We first apply the adjoining cell mapping technique to the well-known van der Pol oscillator described by

$$
\begin{aligned}
& \dot{x}_{1}=x_{2} \\
& \dot{x}_{2}=\mu\left(1-x_{1}^{2}\right) x_{2}-x_{1}
\end{aligned}
$$

For $\mu>0$, this system possesses a single asymptotically stable limit cycle and an unstable equilibrium at the origin. When the simple cell mapping method is applied to this, the results depend on the chosen value for the integration time $T$. In general, as illustrated in [3, 4], the stable limit cycle is represented by several groups of periodic cells in the cell state space. Each group consists of periodic cells which are usually disconnected (that is, nonadjoining). The number of groups and periodic cells depend strongly on the value of $T$.

Figure 1 shows the results of applying adjoining cell mapping method to the system (3) for $\mu=1$ where we have chosen an initial integration time duration $T=0.025$ with step size $\Delta t=T$. The region of interest covers the range $-2.490234375 \leq x_{1}<2.509765625$ and $-2.98828125 \leq x_{2}<$ 3.01171875 with each state variable partitioned into 256 intervals (comprising of a total of 65,536

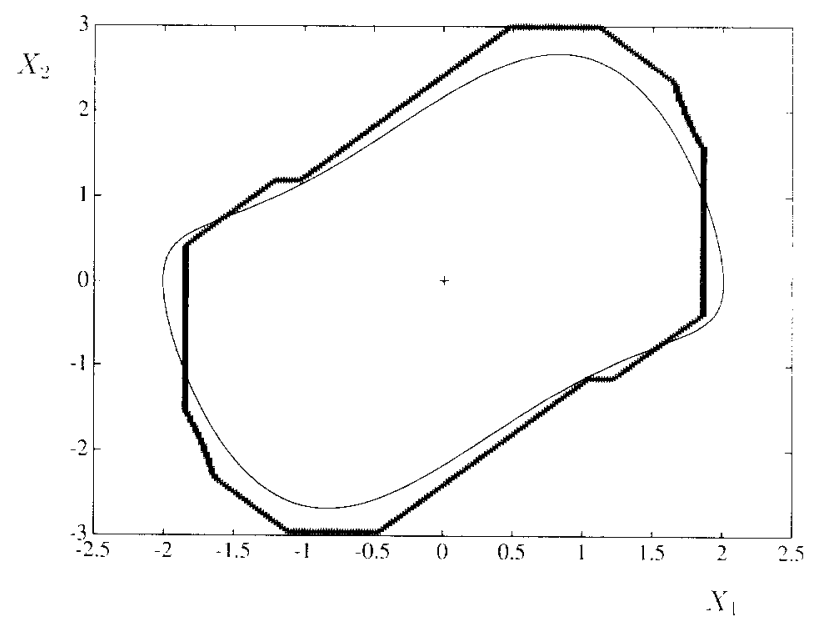

Fig. 1. A single group of periodic cells $(P-592)$ obtained by the adjoining cell mapping method which replaces the limit cycle of van der Pol equation (3) for $\mu=1.0$. 
cells in the cell state space). Note that the origin of state space is represented by a single equilibrium cell denoted by symbol ' + '. The asymptotically stable limit cycle (shown by the solid curve) is characterized by a single group of periodic cells with period 592 (or $P-592$ cells, shown by symbol '*'), and by definition of adjoining mapping, these periodic cells are connected.

For comparison, the results obtained by performing a simple cell mapping analysis of (3) for the same state space region and discretization are summarized in Table I for various values of constant integration duration $T_{S}$ which is employed for the purpose of generating an associated simple cell mapping. Figure 2 shows the periodic cells located by the simple cell mapping

TABLE I.

Representation of the origin and the limit cycle for $\mu=1.0$ for (3) by the simple cell mapping analysis ( $256 \times 256$ cells) for various durations of integration time $T_{S}$

\begin{tabular}{|c|c|c|c|c|}
\hline \multicolumn{2}{|l|}{$\begin{array}{l}\text { Integration } \\
\text { parameters }\end{array}$} & \multirow{2}{*}{$\begin{array}{l}\text { Periodic cells } \\
\text { near origin }\end{array}$} & \multicolumn{2}{|l|}{$\begin{array}{l}\text { Periodic cells } \\
\text { replacing limit cycle }\end{array}$} \\
\hline$T_{s}=1.5$ & $\Delta t=T_{s} / 15$ & & $P-40(3)$ & $P_{T}=120$ \\
\hline$T_{S}=1.0$ & $\Delta t=T_{S} / 10$ & $P-1$ & $P-20(6)$ & $P_{I}=120$ \\
\hline$T_{S}=0.5$ & $\Delta t=T_{S} / 5$ & $P-1$ & P-40 (3) & $P_{T}=120$ \\
\hline$T_{S}=0.25$ & $\Delta t=T_{s} / 5$ & $P-1(15)$ & $P-80$ & $P_{T}=80$ \\
\hline$T_{s}=0.1$ & $\Delta t=T_{S} / 5$ & $P-1(99)$ & $P-67(4)$ & $P_{T}=268$ \\
\hline$T_{S}=0.05$ & $\Delta t=T_{S} / 5$ & $P-1(383)$ & $P-134$ & $P_{T}=134$ \\
\hline$T_{S}=0.025$ & $\Delta t=T_{S} / 5$ & $P-1(1459)$ & $P-258$ & $P_{T}=258$ \\
\hline$T_{S}=0.02$ & $\Delta t=T_{s} / 5$ & $P-1(2398)$ & No $P-K$ cells & \\
\hline$T_{S}=0.01$ & $\Delta t=T_{s} / 5$ & $P-1(13467)$ & No $P-K$ cells & \\
\hline
\end{tabular}

(a)

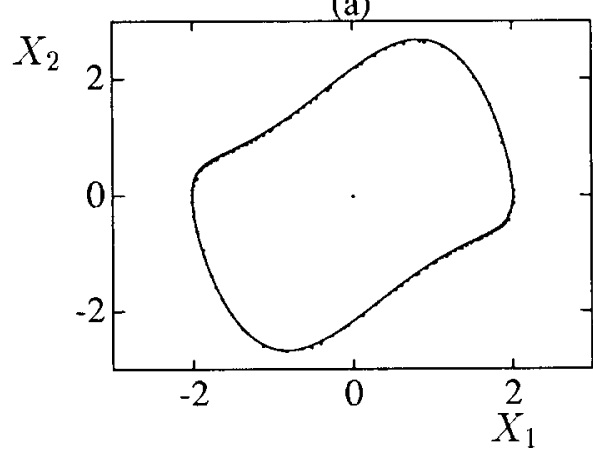

(c)

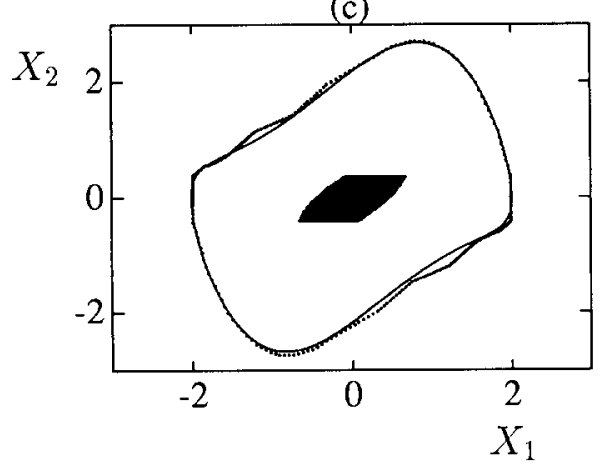

(b)

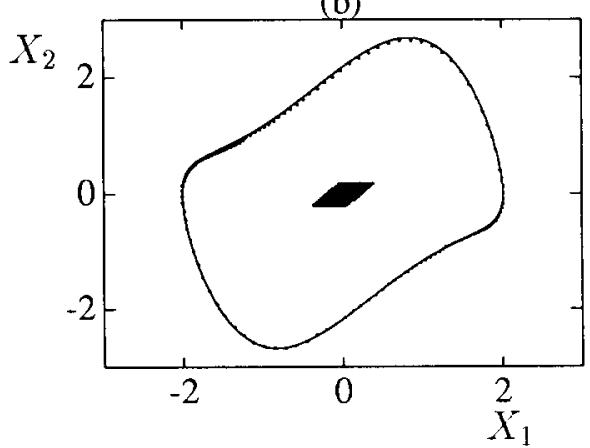

(d)

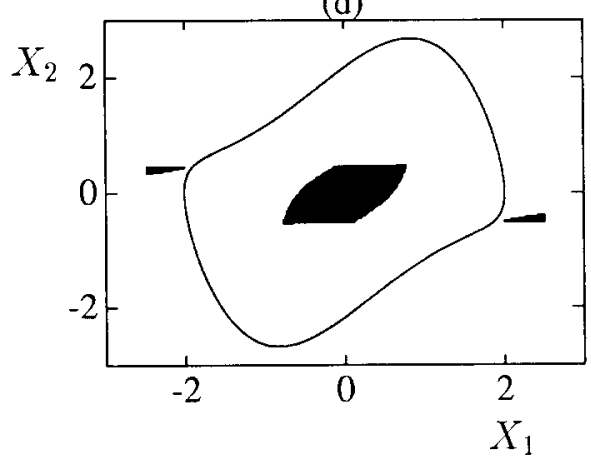

Fig. 2. Simple cell mapping results displaying periodic cells which replace the origin and the limit cycle of van der Pol equation (3) for $\mu=1.0$ with (a) $T_{s}=0.5$, (b) $T_{s}=0.05$, (c) $T_{s}=0.025,(d) T_{s}=0.02$. 
technique for $T_{S}=0.5,0.05,0.025,0.02$. Table I displays the effect of integration duration $T_{S}$ where the notation $P-K$ denotes a periodic motion of period $K \in \mathbf{Z}$ and $P_{T}$ refers to the total number of periodic cells representing the limit cycle. If more than one group of periodic cells with the same periodicity appears, then the number of these groups is indicated in parenthesis. In all the cases, it was found that the stable limit cycle of (3) is replaced by several groups of disconnected periodic cells. In Table I, particularly note the variation in the periodicity of the discovered $P-K$ groups which sporadically cover the limit cycle. An increasingly large number of periodic cells cluster around the origin as $T_{S}$ is made smaller (in this case all the periodic cells are $P-1$ cells). The shape of the limit cycle made up of periodic cells detected by the simple cell mapping analysis begins to distort for $T_{S}<0.05$ and this method is unable to detect the limit cycle for $T_{S}<0.02$ as the last two rows of Table I indicates.

The effect of increasing the number of cells for a fixed region $-2.5 \leq x_{1}<2.5$ and $-3.0 \leq$ $x_{2}<3.0$ is shown in Table II for $\mu=1.0$ where the number of periodic cells replacing the limit cycle is provided. For the simple cell mapping analysis we have used $T_{S}=1.0$ and $\Delta T=T_{S} / 10$ whereas for the adjoining cell mapping an initial $T=0.005$ with $\Delta t=T$ has been employed. As expected, because of the adjoining property, the adjoining mapping technique provides just one group of periodic cells for the limit cycle and the number of periodic cells increases as the number of regular cells in the cell state space increases. Tables I and II indicate a clear advantage of the adjoining mapping method over the simple cell mapping method.

Since the adjoining mapping is not unique, it has been observed that the shape of the periodic group of cells corresponding to the limit cycle may vary depending on the way in which an adjoining mapping is determined. But in all cases, only one single periodic group replacing the limit cycle has been detected.

It should be noted that the discretization error generated by an adjoining mapping is more noticeable when delineating the shape of the limit cycle than that induced by a simple cell mapping. This may be explained as follows. The discretization error of a simple cell mapping is reduced when it is obtained by integrating over a long interval of time for a system which possesses asymptotically stable invariant sets. Since an adjoining mapping only considers trajectories until they transit to an adjoining cell, in general, it will result in a considerably shorter duration of integration time than the one employed for a simple cell mapping analysis. Hence, the discretization error is usually more pronounced.

Considering the system (3), cells in the neighborhood of the limit cycle map via a simple cell mapping into cells which lie precisely on the limit cycle after a long enough interval of time. This

TABLE II.

Representation of the limit cycle for $\mu=1.0$ for (3) by the simple and adjoining cell mapping methods for various cell sizes

\begin{tabular}{|c|c|c|c|}
\hline Number of cells & Simple cell mapping & & Adjoining cell mapping \\
\hline $50 \times 50$ & $P-20$ & $P_{T}=20$ & $P-80$ \\
\hline $100 \times 100$ & $P-20(4)$ & $P_{T}=80$ & $P-160$ \\
\hline $150 \times 150$ & $P-20(4)$ & $P_{T}=80$ & $P-258$ \\
\hline $200 \times 200$ & $P-20(5)$ & $P_{T}=100$ & $P-302$ \\
\hline $250 \times 250$ & $P-20(8)$ & $P_{T}=160$ & $P-474$ \\
\hline $300 \times 300$ & $P-20(10)$, & $P_{T}=200$ & $P-512$ \\
\hline $350 \times 350$ & $P-20(9)$ & $P_{T}=180$ & $P-570$ \\
\hline $400 \times 400$ & $P-20(9)$ & $P_{T}=180$ & $P .606$ \\
\hline $450 \times 450$ & $P-20(12)$ & $P_{T}=240$ & $P-932$ \\
\hline $500 \times 500$ & $P-20(14)$ & $P_{T}=280$ & $P-1054$ \\
\hline
\end{tabular}


may not be the case for an adjoining mapping and is reflected here (see Figure 1) in the distorted shape of the periodic group. As expected, the shape still preserves symmetry present in the original dynamical system. On the other hand, when unstable invariant sets are present, discretization error due to a simple cell mapping can considerably increase during a long time integration. It explains the difficulties of the simple cell mapping method to locate unstable invariant sets. This is not always the case for an adjoining cell mapping which seems to locate unstable invariant sets in many cases because of the fact that it is defined by considering only an adjoining time of integration.

Equation (3) for $\mu<0$ has an unstable limit cycle. Usually, when the simple cell mapping method is applied, this limit cycle is not located. Figure 3 shows the results of applying adjoining cell mapping to (3) with $\mu=-0.5$ where the region $-2.5 \leq x_{1}<2.5$ and $-3.0 \leq x_{2}<3.0$ is partitioned to contain $401 \times 401$ cells with an initial $T=0.1$ and $\Delta T=T$. The adjoining mapping is obtained by using the simplified integration procedure mentioned in Section 3.4 of [11]. The unstable limit cycle is represented by a single group of $P-822$ cells (shown by symbol '*') whereas the origin is represented by two groups consisting of a $P-1$ cell (symbol ' + ') and an adjoining $P-14$ cells (symbol ' $\cdot$ ). The unstable limit cycle has been detected in many instances, although the shape is sometimes quite distorted. It is worth to mention that the simplified procedure seems to provide better results (for example the shape) than the regular integration schemes (fourth-order Runge-Kutta). This can be explained by the fact that an accurately computed trajectory tends to diverge from the unstable limit cycle. The simplified approach, based only on the information of the vector field value at a point (at which it is tangent to the trajectory) provides an adjoining mapping which remains closer to the unstable limit cycle.

Let us now consider the system (3) for $\mu=10$. It is known that both fast and slow motions occur along the stable limit cycle. When the simple cell mapping method is applied in the state space region covering the range $-2.5 \leq x_{1}<2.5$ and $-18.0 \leq x_{2}<18.0$ with $201 \times 201$ cells with $T_{S}=0.25$ and $\Delta t=T_{S} / 10$, several groups of periodic cells (symbol ' $*$ ') are obtained as displayed in Figure 4. It is not possible to conclude that a single stable limit cycle exists by merely observing the numerous periodic groups in Figure 4. Every periodic group must be further examined in detail to ascertain if it is a candidate for a limit cycle. After a detailed examination, it turns out

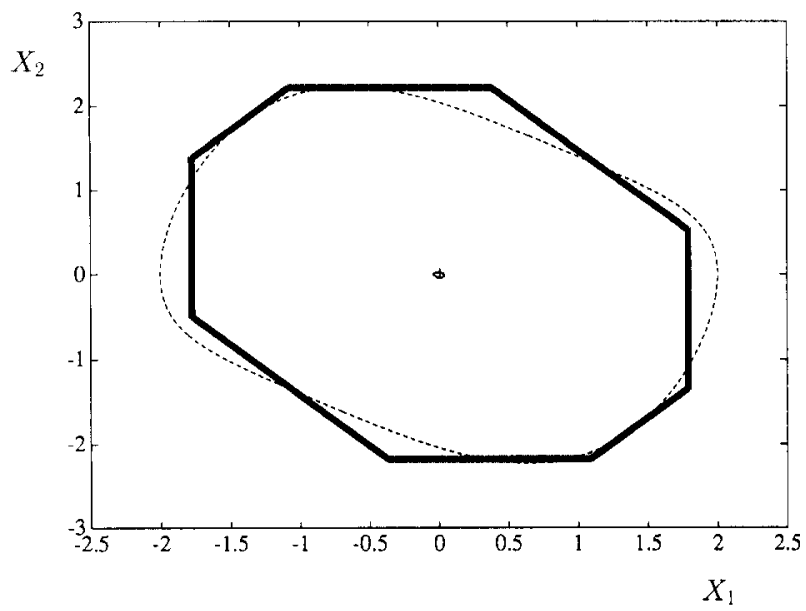

Fig. 3. A single group of periodic cells $(P-822)$ obtained by the adjoining cell mapping method which replaces the unstable limit cycle of van der Pol equation (3) for $\mu=-0.5$. 


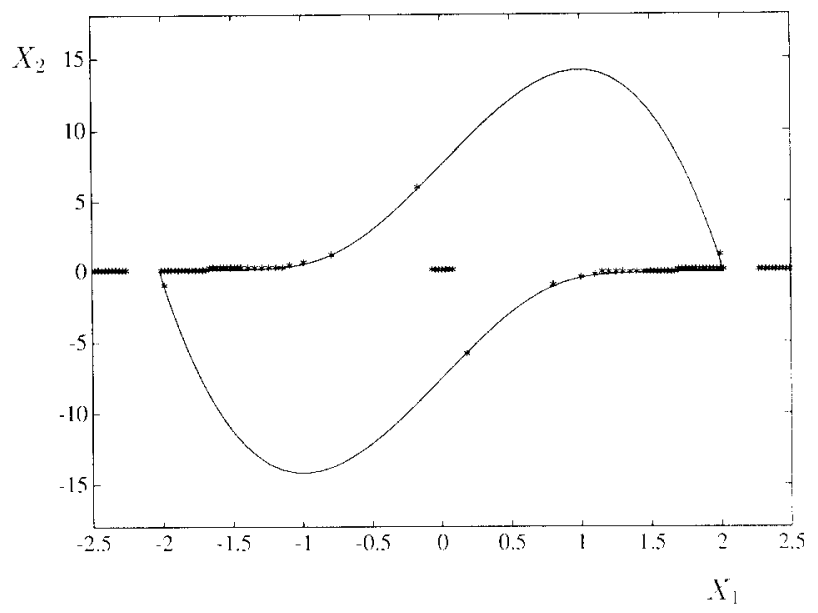

Fig. 4. Several groups of periodic cells obtained by the simple cell mapping method for the van der Pol equation (3) for $\mu=10.0$.

that the limit cycle of (3) is covered by one group of $P-70$ cells and the origin by 7 groups of $P-1$ cells. In addition, there are 2 groups of ten $P$-1 cells which are located along the $x_{1}$-axis. These are pseudo periodic cells and do not provide any useful information. On the other hand, Figure 5 shows the results provided by adjoining cell mapping with an initial $T=0.25$ with $\Delta t=T / 10$. The unstable origin is represented by a $P-1$ cell shown by symbol ' + '. The existing limit cycle is clearly delineated by a unique group of $P$-356 cells (symbol ' $*$ ') which approximately follows the shape corresponding to the true limit cycle of (3). This case illustrates the capability of the new adjoining mapping method in providing improved results over the simple cell mapping method when fast changing dynamics prevails.

\subsection{Recursive Unraveling Algorithm}

We illustrate the algorithm for recursive unraveling of the adjoining cell mapping (developed in Section 4 of [11]) and its memory requirement for practical implementation by considering again

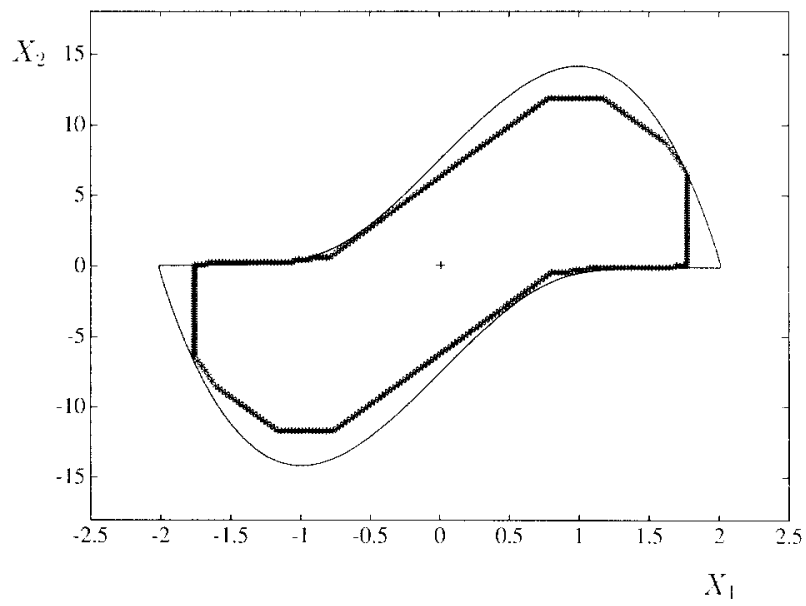

Fig. 5. A single group of periodic cells $(P-356)$ obtained by the adjoining cell mapping method for the van der Pol equation (3) for $\mu=10.0$. 
the van der Pol oscillator (3) with $\mu=1$. Once the simple cell mapping is defined, the unraveling algorithm described in [3] provides periodic cells representing the limit cycle. For that purpose, the algorithm requires to store the entire cell mapping (mapping corresponding to every cell in state space region). Suppose that the state space region under consideration is partitioned into $256 \times 256$ intervals containing a total of 65,536 cells. As required by the simple cell mapping unraveling algorithm, for computational purposes one needs to allocate memory for 65,536 elements to store the cell mapping alone.

On the other hand, let us consider the recursive algorithm which has been implemented as described in Section 4 of [11]. Suppose that each of the state space variables is also partitioned into 256 intervals, the total number of cells amounting also to 65,536 . The region of state space under study is partitioned, in the first level of partition, into 4 blocks by dividing each state variable by half. Each block is partitioned in turn into 4 blocks following the same procedure. The partitioning stops at the level where the blocks are of size 2 by 2 intervals (or 4 cells).

Hence, the lower (first) level of analysis deals with regions of 2 by 2 intervals (that is, only 4 cells). As explained in Section 4 of [11], each block is analyzed independently after finding an adjoining mapping for each of these four cells. When the recursive algorithm is applied to (3), the first level of analysis provides a $P-1$ cell corresponding to the equilibrium at the origin which is shown by symbol ' + ' in Figure 6. Such a cell and the ones that map to it (if any at all) become inactive cells for analysis at further levels. Once four adjoining blocks have been analyzed and the resulting inactive cells discarded, the analysis of the new block formed by the union of these four blocks is performed in the second level of analysis. For that purpose, only the active cells will be kept in memory. Again, notice that each block in this second level of analysis is considered separately and we need to keep in memory the mapping corresponding to the active cells belonging to only one block at a time.

The last level of analysis corresponds to the total state space region under consideration, which in this case provides only 8 active cells (two in each block) to define a periodic solution that characterizes the existing limit cycle with minimum memory requirements. The location of these active cells are shown by symbol 'o' in Figure 6. Finally, as explained in Section 4.3 of [11], once this periodic solution is located, it is straightforward to determine the complete set of correspond-

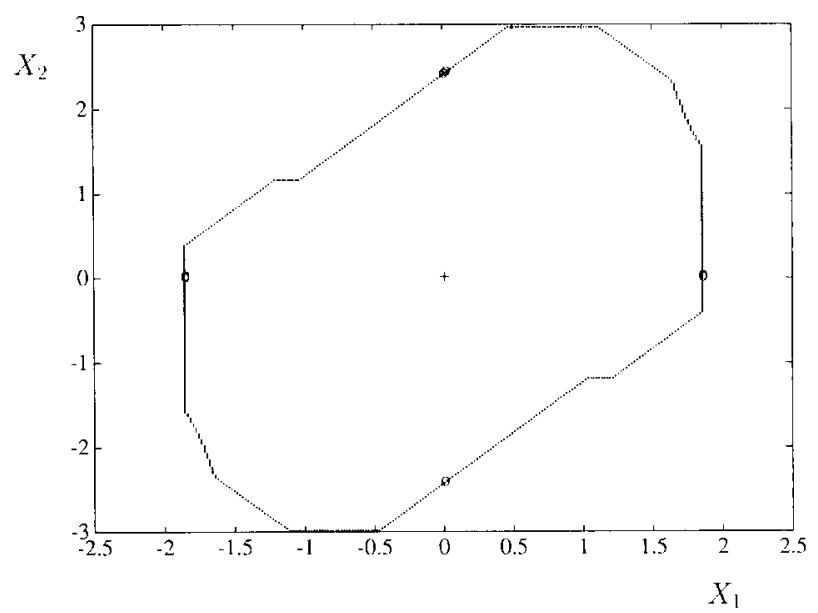

Fig. 6. Completion of the periodic group starting from any one of the active cells (shown by ' $o$ ') to detect the cells covering the limit cycle (shown by '"'). 
ing invariant adjoining cells that constitute the periodic solution. Any one of these 8 active cells can be employed to generate $P-K$ cells which comprises the limit cycle of (3). The complete periodic cells so obtained is shown in Figure 6 by symbol ' ', (These periodic cells are the same as those shown in Figure 1).

The memory management of the present implementation of the recursive unraveling algorithm is dependent on the parameter $E$ presented in equation (13) of [11]. This means that the total number of mappings to be stored is given by

$$
2 \times E \times\left(256 \times 256-2^{2} \times 127 \times 127\right)=2040 E \text {. }
$$

Following the above analysis for the recursive scheme, it is very clear that the maximum memory required is bounded above by 2040 elements. It is worth to mention that our particular implementation of memory management is not the best suited for the analysis of van der Pol oscillator, since most of the jump cells remain active during the whole analysis process. According to our computational results, the recursive unraveling algorithm for (3) needed to store 1940 cell mappings in the last level of analysis ( $E=0.95$, or $95 \%$ of jump cells were active). Nevertheless, the memory employed implies a saving of more than 32 times the memory needed by the standard unraveling algorithm of simple cell mapping provided in [3]. Memory reduction of this order will be a critical factor which will enable the cell mapping method to analyze higher dimensional systems in future.

\subsection{Example 2. Multiple Limit Cycles}

We now consider the following system which possesses infinitely many limit cycles:

$$
\begin{aligned}
& \dot{x}_{1}=x_{2}, \\
& \dot{x}_{2}=-\mu \sin x_{2}-x_{1} .
\end{aligned}
$$

In the state space region defined by $-20 \leq x_{1}<20$ and $-20 \leq x_{2}<20$, this system for $\mu>0$ possesses six concentric limit cycles and an asymptotically stable equilibrium point at the origin. The inner limit cycle is unstable while the rest are alternately asymptotically stable and unstable.

First, we display the simple cell mapping results by partitioning each state variable into 300 intervals (with a total of 90,000 cells in the cell state space) and take $\mu=1.0$. Figure 7 shows the results which corresponds to a constant integration duration $T_{S}=0.5$ units of time with $\Delta t=T_{S} / 8$ while Figure 8 corresponds to a shorter duration of $T_{S}=0.05$ with $\Delta t=T_{S} / 8$. Stable limit cycles are indicated by solid lines, unstable limit cycles by dashed lines and periodic cells by symbol ' $\cdot$ '. The results are tabulated in Table III. Note that none of the two results is satisfactory. For $T_{S}=0.5$ all the unstable limit cycles are missed whereas for $T_{S}=0.05$ two of the unstable limit cycles are missed. In addition, and especially for $T_{S}=0.05$, the periodic cells do not delineate clearly the true limit cycles and the equilibrium at the origin is surrounded by a very large set of $P-1$ cells. Referring to Figure 7 , the periodic cells which represent the limit cycle $S_{1}$ distorts its shape slightly, a large number of periodic groups represent $S_{2}$ forming a thick band around it, and the periodic cells representing $S_{3}$ are not in its close proximity. As evidenced in Figure 8 , larger integration durations usually provide a 'cleaner' set of results for the simple cell mapping method with a smaller number of periodic cells representing the limit cycle. 


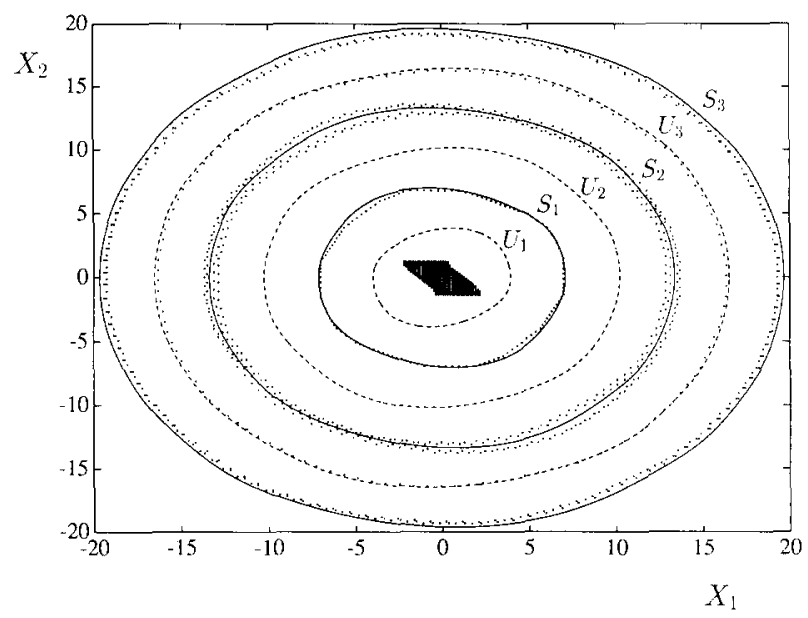

Fig. 7. Simple cell mapping results for (4) with $T_{S}=0.05$. Several groups of $P-K$ cells replace each limit cycle and two unstable limit cycles are missed.

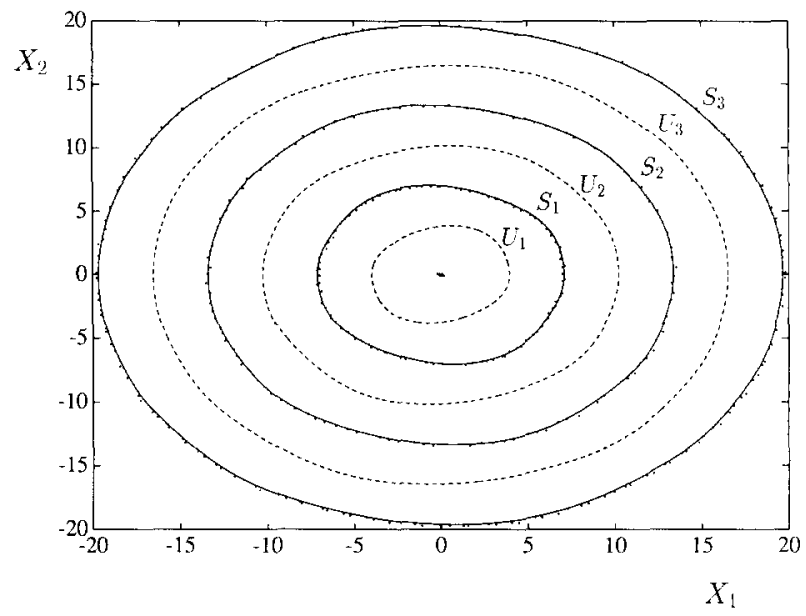

Fig. 8. Simple cell mapping results for (4) with $T_{S}=0.5$. Several groups of $P-K$ cells replace each limit cycle and all the three unstable limit cycles are missed.

TABLE III.

Representation of the origin and the limit cycles for $\mu=1.0$ for (4) by the simple and adjoining cell mapping methods

\begin{tabular}{llll}
\hline Invariant & Simple cell mapping & Simple cell mapping & \multicolumn{1}{c}{$\begin{array}{l}\text { Adjoining cell } \\
\text { mapping }\end{array}$} \\
\hline sets & $T=0.05$ & $T=0.5$ & $P-1(4)$ \\
Origin & $P-1(412)$ & $P-6(6)$ & $P-146$ \\
Limit cycle $U_{1}$ & No $P$ - $K$ cells & No $P-K$ cells & $P-266$ \\
Limit cycle $S_{1}$ & $P-128$ & $P-38(3)$ & $P-396$ \\
Limit cycle $U_{2}$ & No $P$ - $K$ cells & No $P$ - $K$ cells & $P-534$ \\
Limit cycle $S_{2}$ & $P-126(4)$ & $P-63(2)$ & $P-762$ \\
Limit cycle $U_{3}$ & $P-126$ & No $P-K$ cells & $P-890$ \\
Limit cycle $S_{3}$ & $P-126(3)$ & $P-88(2)$ & \\
\hline
\end{tabular}


For the same cellular state space with an initial $T=0.05$ and $\Delta t=T / 6$, the results of adjoining mapping method appear in Figure 9. Note that the adjoining mapping locates all the six limit cycles and the equilibrium point at the origin (shown by symbol ' $'$ ). Each limit cycle is characterized by a single group of periodic cells shown by symbol ' *'. Moreover, as the size of the limit cycle increases, the new method provides larger $P$ - $K$ groups which is to be expected because of the adjoining property of the map. It is satisfying to observe that the adjoining mapping technique is able to perform much better than the simple cell mapping method in locating all the stable and unstable limit cycles which are usually missed while carrying out numerical computations.

\subsection{Example 3. Limit Cycle in $R^{3}$}

The following vector field $\mathbf{F}$ in $\mathbf{R}^{3}$ is taken from [1]:

$$
\begin{aligned}
& F_{1}=-c x_{1}+x_{2}-\phi\left(x_{1}\right), \\
& F_{2}=-x_{1}+x_{3}, \\
& F_{3}=-c x_{1}+b \phi\left(x_{1}\right) .
\end{aligned}
$$

Here $b>0, c>0, b<c^{2}$ and $\phi\left(x_{1}\right)$ is given by

$$
\phi\left(x_{1}\right)= \begin{cases}1.6\left|x_{1}\right|, & \left|x_{1}\right|<a \\ \left(-20\left|x_{1}\right|+502\right) \operatorname{sgn} x_{1}, & a<\left|x_{1}\right|<25 \\ \left(0.15\left|x_{1}\right|-1.75\right) \operatorname{sgn} x_{1}, & \left|x_{1}\right|>25\end{cases}
$$

where $a=502 / 21.6=23.240740407$. The function $\phi\left(x_{1}\right)$ is chosen to represent a nonlinearity in the sector $(0, k), k>1.6$ and satisfies $\phi(0)=0$ and $0<\phi\left(x_{1}\right) / x_{1}<k$ for all values of $x_{1} \neq 0$. We choose $b=0.5$ and $c=1.0$. For this set of parameter values, the dynamical system (1) with $\mathbf{F}$ given by (5) possesses an asymptotically stable equilibrium point located at the origin $\mathbf{x}^{*}=(0,0$, 0 ) and an asymptotically stable limit cycle, for more details see [1].

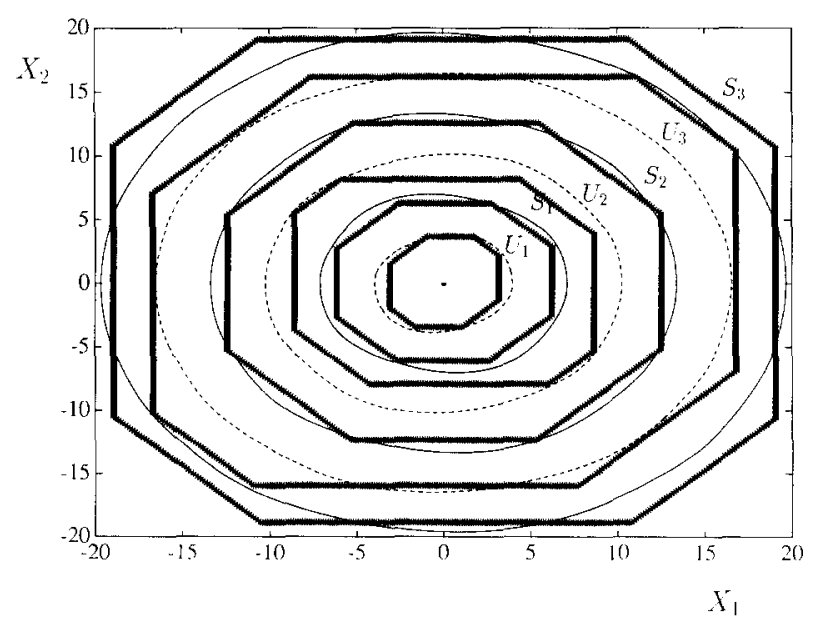

Fig. 9. Adjoining cell mapping results for (4). Each limit cycle is replaced by a single group of $P$ - $K$ cells. All the three unstable limit cycles are detected. 
Simple cell mapping method is applied to the region $-60 \leq x_{1}<60,-100 \leq x_{2}<100$ and $-60 \leq x_{3}<60$ where each state variable is partitioned into 201 intervals (a total of $8,120,601$ cells) with $T_{S}=0.75$ and $\Delta t=T_{S} / 25$. The projection of location of the periodic cells found by the simple cell mapping on the $x_{1}-x_{2}, x_{1}-x_{3}$, and $x_{2}-x_{3}$ planes are shown in Figures 10(a)-(c) and the results are tabulated in Table IV. The periodic cells corresponding to the limit cycle and $\mathbf{x}^{*}$ are represented by symbol "*" and ".", respectively. The limit cycle of the dynamical systems is replaced by three groups of $P-60, P-14$ and $P-12$ cells. Our study has shown that the simple cell

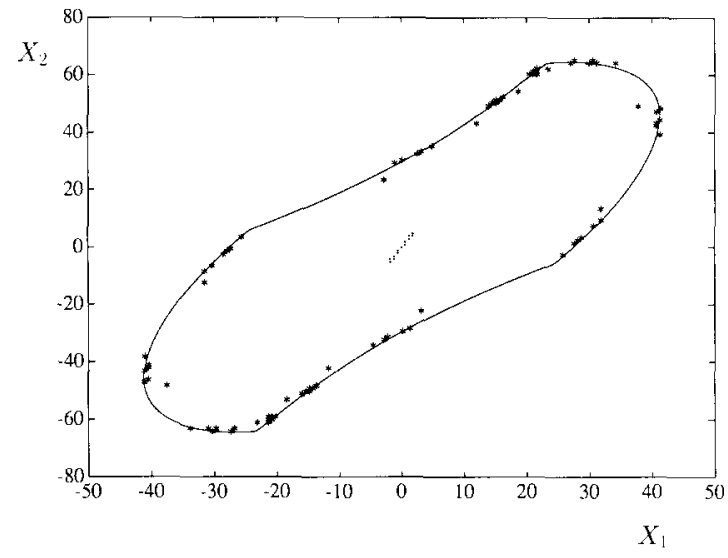

(a)

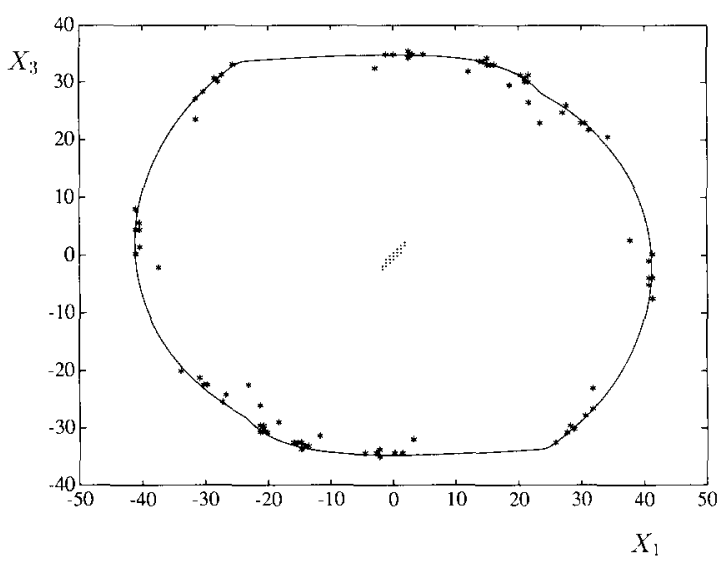

(b)

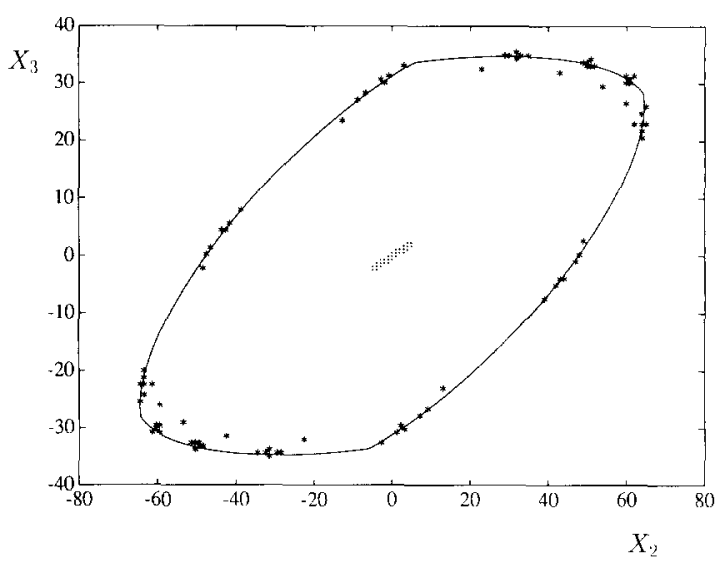

(c)

Fig. 10. Location of periodic cells detected by the simple cell mapping method for Example 3 .

TABLE IV.

Periodic cells detected by the simple and adjoining cell mapping methods in the cellular state space for Example 3

\begin{tabular}{ll}
\hline Method & Representation of invariant groups \\
\hline Simple cell mapping & $\mathbf{x}^{*}: P-1(29)$ \\
& Limit cycle: $P-60, P-14, P-12 ; P r=86$ \\
Adjoining cell mapping & $\mathbf{x}^{*}: P-1(1), P-2(4)$ \\
& Limit cycle: $P-398$ \\
\hline
\end{tabular}


mapping method generates periodic cells whose proximity to the limit cycle depends on the cell size, the duration of integration $T_{S}$, and the accuracy of integration. The number of periodic cells also vary widely depending on the same factors (these are the reasons for choosing the value $T_{S}=0.75$ and a small time-step $\Delta t$ for this problem after a long experimentation). As expected, the location of periodic cells has been observed to be closer to the limit cycle for large values of $T_{S}$ with very accurate integration schemes.

The adjoining cell mapping method is applied to the same region with an initial $T=0.0075$ and $\Delta t=T / 12$. This method provides a single group of connected $P$-398 cells associated with the true limit cycle as shown in Figures 11 (a)-(c). This example demonstrates the capability of the adjoining cell mapping method in providing a qualitative behavior of higher order dynamical systems.

\subsection{Example 4. A Hamiltonian System}

The determination of invariant sets for Hamiltonian systems is quite involved since their trajectories are volume preserving. In this section, we will compare the simple and adjoining cell

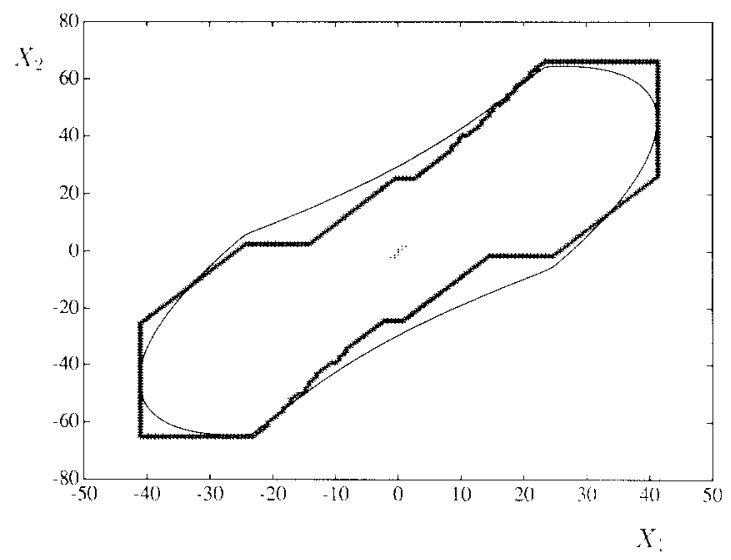

(a)

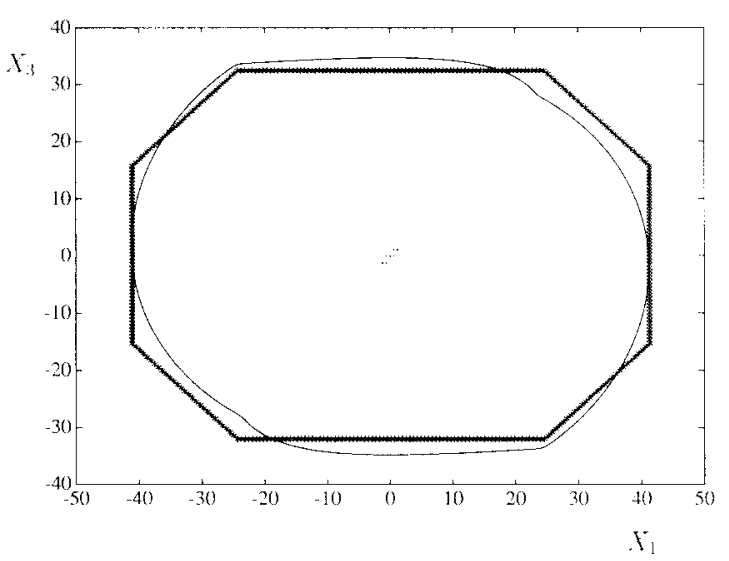

(b)

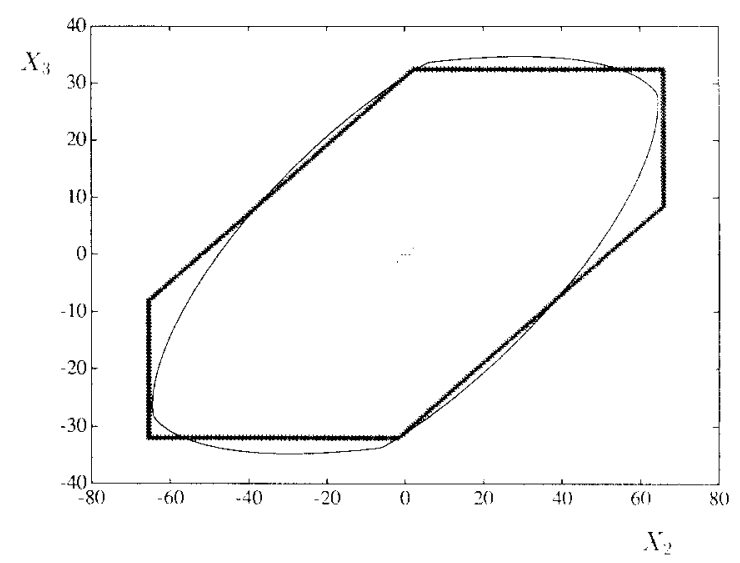

(c)

Fig. 11. Location of periodic cells detected by the adjoining cell mapping method for Example 3 . 
mapping methods by applying them to a Hamiltonian system processing infinite nonisolated periodic solutions. Trajectories starting close to one another initially will not approach each other (and, typically, do not even remain nearby) as $t \rightarrow \infty$ no matter how close they are. The cell mapping methods presume that by considering a single trajectory in a given cell, the long term behavior of all trajectories initiating nearby or within this cell can be determined and is characterized by a single entity (the mapping of this cell). This basic assumption is not satisfied by Hamiltonian systems. Therefore, one can expect that a discretization applied to state space can produce a severe distortion of the original dynamics.

For an analysis of a Hamiltonian system, we consider a simple pendulum governed by

$$
\begin{aligned}
& \dot{x}_{1}=x_{2}, \\
& \dot{x}_{2}=-\sin x_{1} .
\end{aligned}
$$

Simple and adjoining cell mapping methods are applied to the region $-4 \leq x_{1}<4$ and $-3 \leq x_{2}<3$ and two different cell sizes are considered for which each state variable is partitioned into (a) 201 intervals (or 40,401 cells) and (b) 401 intervals (or 160,801 cells). The simple cell mapping results with $T_{s}=1$ and $\Delta t=T_{s} / 6$ are shown in Figures 12(a)-(b). The solid line corresponds to the homoclinic orbit originating at the saddle point of (6). The results indicate that a large number of periodic groups correspond to the infinite periodic solutions possessed by (6), see Table $\mathrm{V}$ where $G_{T}$ refers to the total number of $P-K$ groups discovered. The shapes of the periodic groups detected by the simple cell mapping analysis define accurately the evolution of periodic solutions in state space but their number and relative location are very confusing. A blank space in Figures 12(a)-(b) indicates that the corresponding cell is either attracted to a periodic group or maps to the sink cell. The effect of state-space discretization is obvious in that the Hamiltonian nature of the dynamics is not preserved since some $P-K$ groups attract nearby cells.

The adjoining cell mapping results with an initial $T=0.05$ and $\Delta t=T / 6$ are presented in Figures 13(a)-(b). This method provides less number of periodic groups with distorted shape but their relative location clearly delineates the fact that the true periodic solutions of (6) are

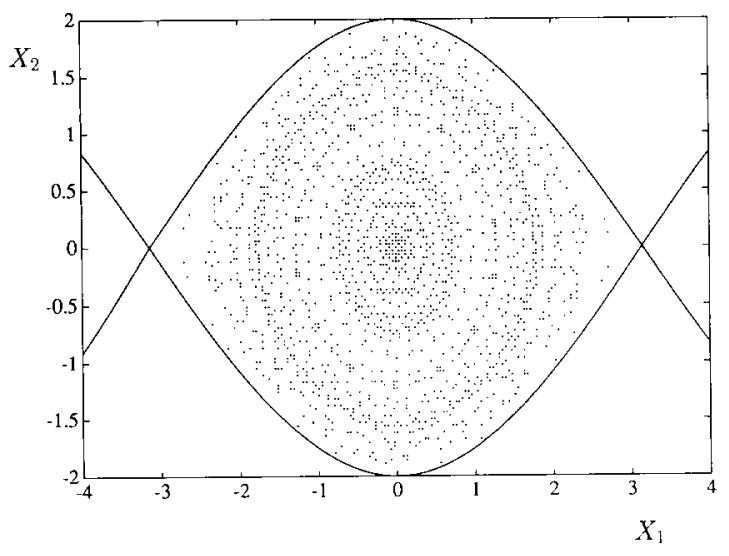

(a)

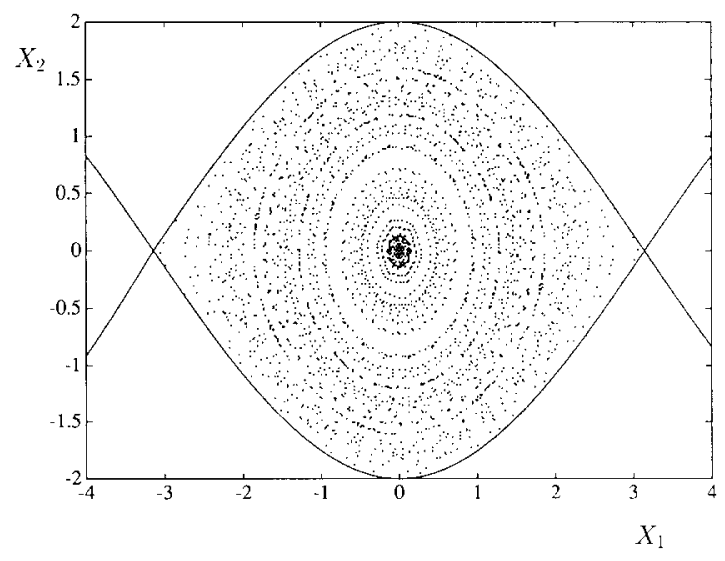

(b)

Fig. 12. Location of periodic cells detected by the simple cell mapping method for the Hamiltonian system (6) with (a) $201 \times 201$ cells, (b) $401 \times 401$ cells. 
TABLE V.

Periodic motions detected by the simple and adjoining cell mapping methods in the cellular state space for the Hamiltonian system (6)

\begin{tabular}{|c|c|}
\hline Cells & $P \cdot K$ cells detected by the simple cell mapping method \\
\hline $201 \times 201$ & $\begin{array}{l}P-86(2), P-78, P-72, P-58, P-51(2), P-48, P-46, P-38(2), P-37(2), P-32(2), \\
P-31(4), P-29(4), P-28(2), P-22, P-20(3), P-14(2), P-13(2), P-12(2), P-10 \\
(3), P-8(11), P-7(16), P-6(14), P-1(3) \\
G_{T}=82, P_{T}=1565\end{array}$ \\
\hline $401 \times 401$ & $\begin{array}{l}P-100(2), P-94, P-88, P-80, P-58, P-50(2), P-48(3), P-46, P-44(2), P-42, \\
P-36, P-37(2), P-34, P-32(5), P-30(2), P-29(2), P-28, P-27(2), P-26(2) \\
P-25(2), P-24(2), P-22(3), P-20(8), P-19(6), P-18, P-17(2), P-14(6), P-13 \\
(6), P-12(4), P-11(4), P-10(9), P-9(2), P-8(23), P-7(16), P-6(14), P-1 \\
G_{r}=142, P P_{T}=2729\end{array}$ \\
\hline Cells & $P-K$ cells detected by the adjoining cell mapping method \\
\hline $201 \times 201$ & $\begin{array}{l}P-324, P-320, P-316, P-312, P-308, P-304, P-300, P-296, P-292, P-288, P-284, \\
P-280, P-276, P-272, P-268, P-264, P-260, P-256, P-252, P-248, P-244, P-240, \\
P-236, P-232, P-228, P-144, P-140, P-136, P-132, P-128 . P-124, P-120, P-116, \\
P-56, P-52, P-48, P-12, P-1 \\
G_{T}=38, P P_{T}=8109\end{array}$ \\
\hline $401 \times 401$ & $\begin{array}{l}P-640, P-636, P-632, P-628, P-624, P-620, P-616, P-612, P-608, P-604, P-600, \\
P-596, P-592, P-588, P-584, P-580, P-576, P-572, P-568, P-564, P-560, P-556, \\
P-552, P-548, P-544, P-540, P-536, P-532, P-528, P-524, P-520, P-516, P-512, \\
P-508, P-504, P-500, P-496, P-492, P-488, P-484, P-480, P-476, P-472, P-468, \\
P-464, P-460, P-456, P-452, P-292, P-288, P-284, P-280, P-276, P-272, P-268, \\
P-264, P-260, P-256, P-252, P-248, P-244, P-240, P-236, P-232, P-228, P-224, \\
P-196, P-136, P-132, P-56, P-52, P-48, P-12, P-1 \\
G_{7}=74, P P_{T}=31485\end{array}$ \\
\hline
\end{tabular}

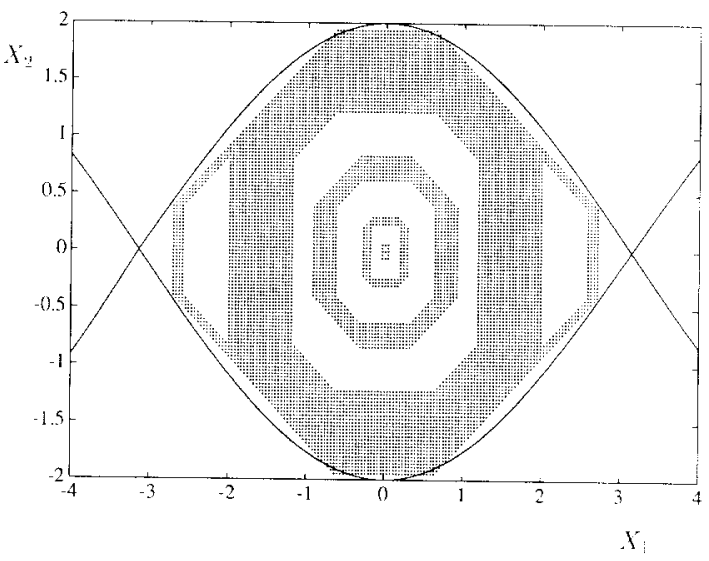

(a)

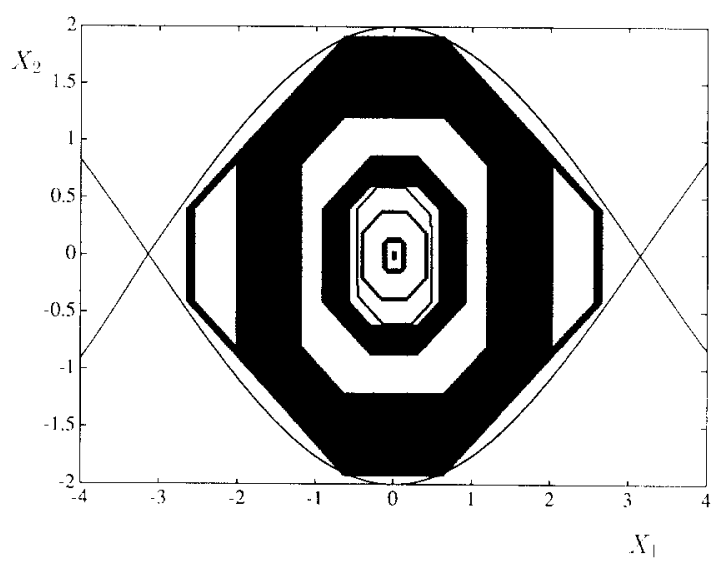

(b)

Fig. 13. (a) Location of periodic cells detected by the adjoining cell mapping method for the Hamiltonian system (6) with (a) $201 \times 201$ cells, (b) $401 \times 401$ cells. 
concentric. Table $\mathrm{V}$ indicates that the adjoining mapping method has detected more than 5 times the number of periodic cells found by the simple cell mapping method. Also, as expected in the new technique, the outer orbits have an increasingly larger number of periodic cells. We have noticed that the periodicity of the $P-K$ group corresponding to the outermost orbit increases as the cell size is decreased. It is worth to mention that the appearance of several spatially close groups of periodic cells (as a matter of fact touching or adjoining groups) is uncommon for an adjoining mapping. Therefore, they may suggest that the dynamical system under study possesses a very large number of periodic solutions and that it may be a conservative system (as it is the case here). Nevertheless, the conclusions must be very carefully drawn.

\subsection{Example 5. Chaotic System}

For a system undergoing chaotic motion, the region of state space attracted by the chaotic attractor (that is, its domain of attraction) evolves in the same way as in the case of a standard dissipative dynamical system. Therefore, cell mapping methods are able to approximately delineate their behavior. This implies that an approximate shape of a chaotic attractor can be determined via cell mapping methods which can then be refined by using the generalized cell mapping method, for details see [4]. On the other hand, concerning the dynamics within the chaotic attractor, these state space discretization procedures present problems similar to the ones mentioned earlier for Hamiltonian systems. Trajectories starting with very close initial conditions will exponentially diverge as $t \rightarrow \infty$ no matter how close they initially are. This again does not fit the basic assumption of cell mapping methods. Therefore, one can expect that a discretization applied to state space in which a chaotic attractor exists will produce results which may need to be interpreted appropriately when relating them to original dynamics.

To illustrate this, we will consider the Lorenz system of equations given by

$$
\begin{aligned}
& \dot{x}_{1}=\sigma\left(x_{2}-x_{1}\right), \\
& \dot{x}_{2}=r x_{1}-x_{2}-x_{1} x_{3}, \\
& \dot{x}_{3}=x_{1} x_{2}-b x_{3} .
\end{aligned}
$$

We choose $\sigma=10, b=\frac{8}{3}$ and $r=28$ for which it is well known that no periodic solutions exist and a chaotic attractor and three unstable equilibria at $\mathbf{x}_{1}^{*}=(0,0,0), \mathbf{x}_{2}^{*}=(6 \sqrt{2}, 6 \sqrt{2}, 27)$, and $\mathbf{x}_{3}^{*}=(-6 \sqrt{2},-6 \sqrt{2}, 27)$ are present, see $[5,7]$.

Simple and adjoining cell mapping methods are applied to the region $-40 \leq x_{1}<40$, $-40 \leq x_{2}<40$ and $-10 \leq x_{3}<80$ where each state variable is partitioned into 200 intervals (a total of $8,000,000$ cells). The result of the simple cell mapping method obtained by taking $T_{S}=0.6$ and $\Delta t=T_{S} / 15$ is presented in Figures 14(a)-(c) and in Table VI. The projection of location of the periodic cells on the $x_{1}-x_{2}, x_{1}-x_{3}$, and $x_{2}-x_{3}$ planes are shown where the solid line corresponds to the chaotic attractor. It is not possible to ascertain which $P$ - $K$ groups belong to which invariant set by merely looking at the periodic cells. Through a careful examination, the periodic groups found by the simple cell mapping method can be identified as shown in Table VI where the quantity $P_{C}$ refers to the total number of periodic cells representing the chaotic attractor. There is a cluster of $25 P$-1 cells near each unstable equilibria $\mathbf{x}_{2}^{*}$ and $\mathbf{x}_{3}^{*}$ and only one $P-1$ cell has been identified to represent each of them. Symbol ' $*$ ' in Figure 14 corresponds to the periodic cells covering the chaotic attractor, ' + ' to $x_{2}^{*}$, ' $x^{\prime}$ ' to $x_{3}^{*}$, and '.' to the unidentifiable 


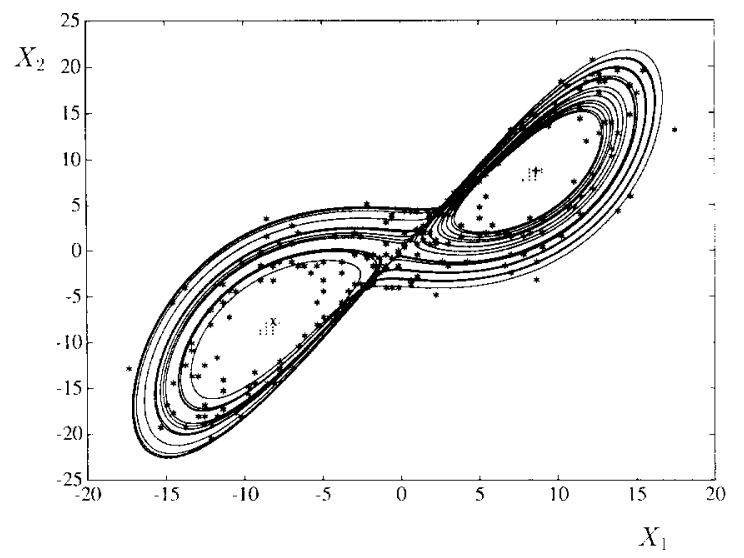

(a)

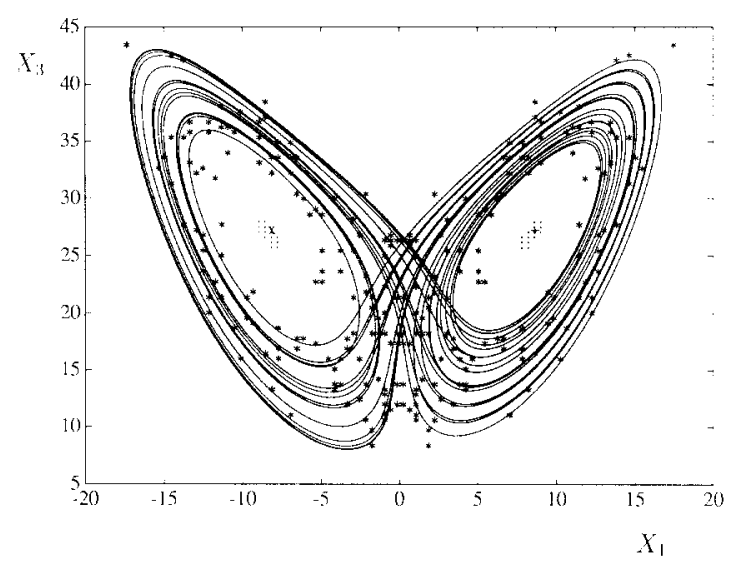

(b)

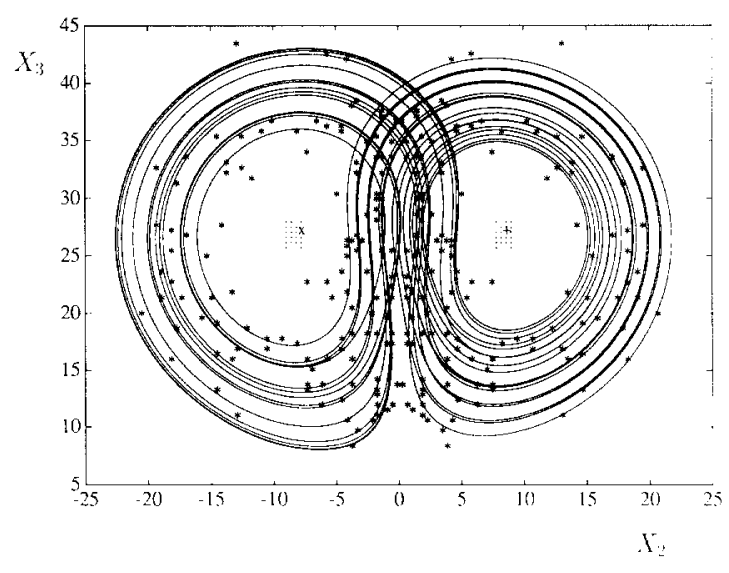

(c)

Fig. 14. Location of periodic cells detected by the simple cell mapping method for the Lorenz system (7).

TABLE VI.

Periodic cells detected by the simple and adjoining cell mapping methods in the cellular state space for the Lorenz system (7)

\begin{tabular}{ll}
\hline Method & Representation of invariant groups \\
\hline Simple cell mapping & $\mathbf{x}_{1}^{*}:$ No $P-K$ cell \\
& $\mathbf{x}_{2}^{*}: P-1, P-1(24)$ \\
& $\mathbf{x}_{3}^{*}: P-1, P-1(24)$ \\
& Chaotic attractor: $P-100, P-50, P-24(2), P-18(2), P-9(2) ;$ \\
& $P_{C}=252$ \\
& $\mathbf{x}_{1}^{*}:$ No $P-K$ cell \\
& $\mathbf{x}_{2}^{*}: P-6, P-24, P-17, P-12$ \\
Adjoining cell mapping & $\mathbf{x}_{3}^{*}: P-6, P-24, P-17, P-12$ \\
& Chaotic attractor: $P-400, P-105(2) ; P_{C}=610$ \\
\hline
\end{tabular}

cluster of $24 P-1$ cells; there is no $P-K$ cell corresponding to the unstable equilibrium point $\mathbf{x}_{1}^{*}$. Note that the location of periodic cells delineate the shape of the chaotic attractor to some extent, but as usual this method provides several periodic groups sporadically placed within the attractor so that the results must be carefully interpreted. 
The adjoining cell mapping method is applied to the same cellular structure with an initial $T=0.01$ and $\Delta t=T / 5$. The results are shown in Figures 15(a)-(c) from which it is easier to identify various periodic groups detected by the method and are depicted in Table VI. The symbols used to represent the $P-K$ cells are the same as those used in Figures 14(a)-(c). The adjoining mapping method results in period motions whose shape in state space is more distorted than that provided by the simple cell mapping. However, it provides only two periodic groups of cells indicating the existence of an attractor whose physical location is in the proximity of the chaotic attractor possessed by (7). The larger group $P-400$ is seen to account for the outermost portion of the attractor while the two smaller groups $P-105$ for each of the two innermost portions. There are six more groups ( $P-24, P-17$ and $P-12$; one set for each unstable equilibria $\mathbf{x}_{2}^{*}$ and $\mathbf{x}_{3}^{*}$ ) which surround the two unstable equilibria. They seem to represent apparent slow motion around them. As before, results of this nature which indicate the presence of several groups of interlaced periodic cells (that are uncommon for adjoining mapping method), perhaps suggest the existence of dynamics interesting and significant enough to warrant further analysis by more sophisticated methods, for example the generalized cell mapping method [4].

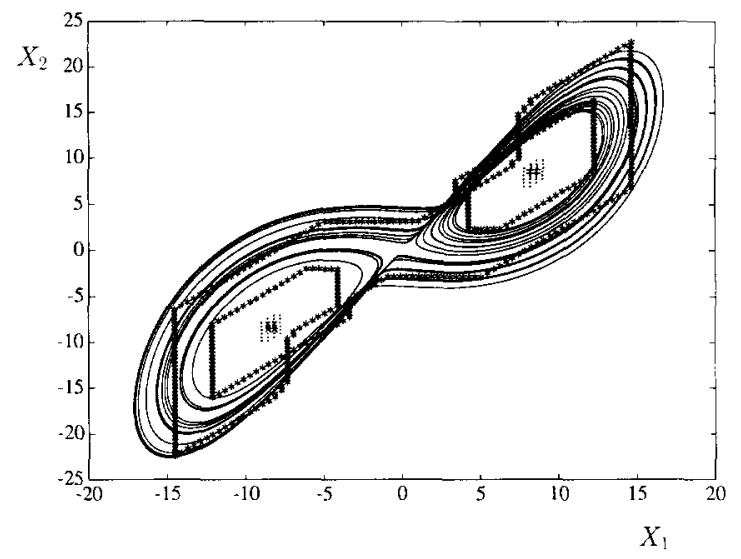

(a)

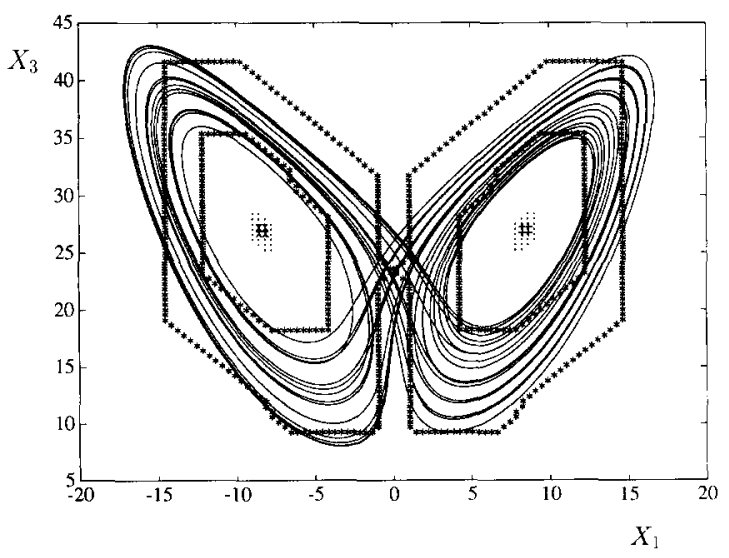

(b)

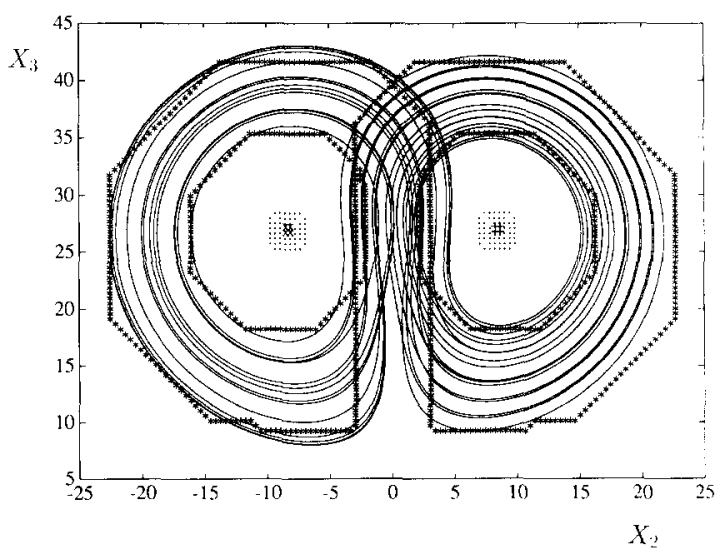

(c)

Fig. 15. Location of periodic cells detected by the adjoining cell mapping method for the Lorenz system (7). 


\section{Application to Nonstandard Dynamical Systems}

In this section, we will illustrate the capability of the adjoining cell mapping method to unravel the global behavior of pathological dynamical systems. Such dynamical systems have been constructed for the purpose of locating all the roots of a nonlinear vector function $f(x)$. The vector field $\mathbf{F}$ of the dynamical system (1) in this case is given by

$$
\mathbf{F}(\mathbf{x})=-\mathbf{J}^{-1}(\mathbf{x}) \mathbf{f}(\mathbf{x}), \quad \mathbf{J}(\mathbf{x})=\nabla_{\mathbf{x}} \mathbf{f}(\mathbf{x})
$$

where $\mathbf{J}$ is the gradient of the vector function $\mathbf{f}$. Analytical and computational studies of these pathological dynamical systems are provided in $[2,8,9,10]$. The norm of $\mathbf{F}$ here may be unbounded on the singular manifolds (manifolds where det $\mathbf{J}(\mathbf{x})=0$ ). One of the major difficulties encountered while numerically analyzing pathological dynamics is how to handle rapid changes of the vector field $\mathbf{F}$ given by (8) for the dynamical system (1). Since the trajectories in the close vicinity of singular manifolds tend to evolve very fast, the traditional computational techniques may not work. The simple cell mapping algorithm integrates trajectories starting in each cell over a fixed duration of time when calculating its mapping. Hence, above mentioned dynamical systems may generate very big 'jumps' in the mapping. The adjoining cell mapping method avoids this problem by automatically adjusting the integration duration to be small whenever needed so that the mapping is always from a cell to an adjoining cell. It seems that the concept of adjoining mapping is helpful in providing a suitable computational tool for analyzing fast dynamics.

We provide two examples with fast and pathological dynamics to illustrate the applicability of the adjoining cell mapping technique. As before, an adjoining cell mapping for these examples is obtained via a fourth-order Runge-Kutta method.

\subsection{Example 6}

Consider the vector function $\mathbf{f}$ in $\mathbf{R}^{2}$ given in [8]:

$$
\begin{aligned}
& f_{1}=x_{1}-4 x_{2}^{2}, \\
& f_{2}=\cos x_{1}-x_{2} .
\end{aligned}
$$

The corresponding vector field $\mathbf{F}$ for (1) is given by

$$
\begin{gathered}
\mathbf{F}(\mathbf{x})=\frac{-1}{\operatorname{det} \mathbf{J}(\mathbf{x})}\left[\begin{array}{c}
-x_{1}+4 x_{2}^{2}+8 x_{2}\left(\cos x_{1}-x_{2}\right) \\
\left(x_{1}-4 x_{2}^{2}\right) \sin x_{1}+\cos x_{1}-x_{2}
\end{array}\right], \\
\operatorname{det} \mathbf{J}(\mathbf{x})=-\left(1+8 x_{2} \sin x_{1}\right) .
\end{gathered}
$$

The roots of $f$ are given by

$$
\begin{aligned}
& \mathbf{x}_{a}^{*}=(1.0366739,0.5090859), \\
& \mathbf{x}_{b}^{*}=(2.4764680,-0.7868399), \\
& \mathbf{x}_{c}^{*}=(3.5021474,-0.9357013),
\end{aligned}
$$


which are all regular roots. They also are the only equilibrium points of (1) and are asymptotically stable. There are not other periodic solutions of (1).

We first apply the method of simple cell mapping to the region $-2.0 \leq x_{1}<8.0$ and $-1.5 \leq x_{2}<1.5$ containing $200 \times 200$ intervals $(40,000$ cells $)$ with $T_{S}=3$ and an integration step size $\Delta t=T_{s} / 6$. The results indicate that there are three isolated $P-1$ cells, one for each of the equilibrium points. In addition, a group of $P-4$ cells near one of the barrier manifolds ${ }^{1}$ (shown by solid lines) is detected. They are fake periodic cells and do not correspond with the equilibria or periodic solutions of the dynamical system under consideration. The location of all the periodic cells are shown in Figure 16(a), the symbol ' ' representing $\mathbf{x}_{a}^{*}$, ' + ' representing $\mathbf{x}_{b}^{*}$, ' $x$ ' representing $\mathbf{x}_{c}^{*}$, '*' representing fake $P-4$ cells, respectively. The fake group of periodic cells corresponds to the point $\mathbf{x}_{S}^{(4)}=(6.1822659,1.2407157)$ in Figure 2 of [9] which is the local minimum of the Lyapunov function $V(\mathbf{x})=\mathbf{f}(\mathbf{x})^{T} \mathbf{f}(\mathbf{x})$ (but not a root of $\mathbf{f}$ ) for the system (1). For more details, the reader is referred to [10]. Figure 16(b) displays the domains of attraction of the equilibrium cells corresponding to (10). Cells represented by symbol "*" are attracted to the group of fake periodic cells. Cells represented by a blank space map to the sink cell. According to the theory for nonstandard dynamical systems provided in [8, 9], trajectories of (1) cannot cross the barrier manifolds. The simple cell mapping results displayed in Figure 16(b) indicate that the cell trajectories of a large number of cells seem to do so. That is why domains of attraction of equilibria of the dynamical system under consideration are composed of disconnected cells.

The adjoining cell mapping method is applied to this example with the same cellular structure. An initial integration time of $T=3$ units $\Delta t=T / 6$ is used to generate an associated adjoining cell mapping of the dynamical system. The equilibrium cells obtained with the new scheme, shown in Figure 17(a), are the same as those obtained earlier by the simple cell mapping method. There are altogether 58 groups of fake $P-2$ cells (more than those shown in Figure 16(a)), each consisting of two adjoining cells (shown by symbol "*") lying close to some of the singular

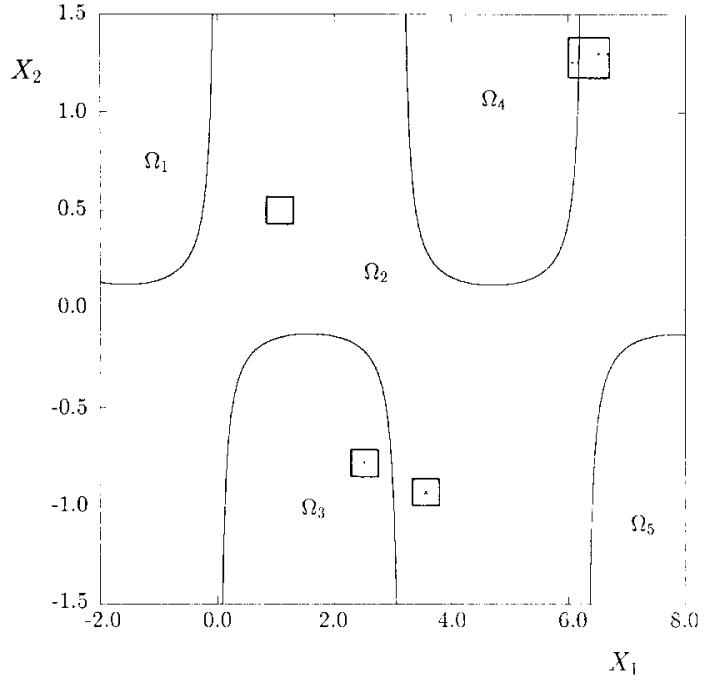

(a)

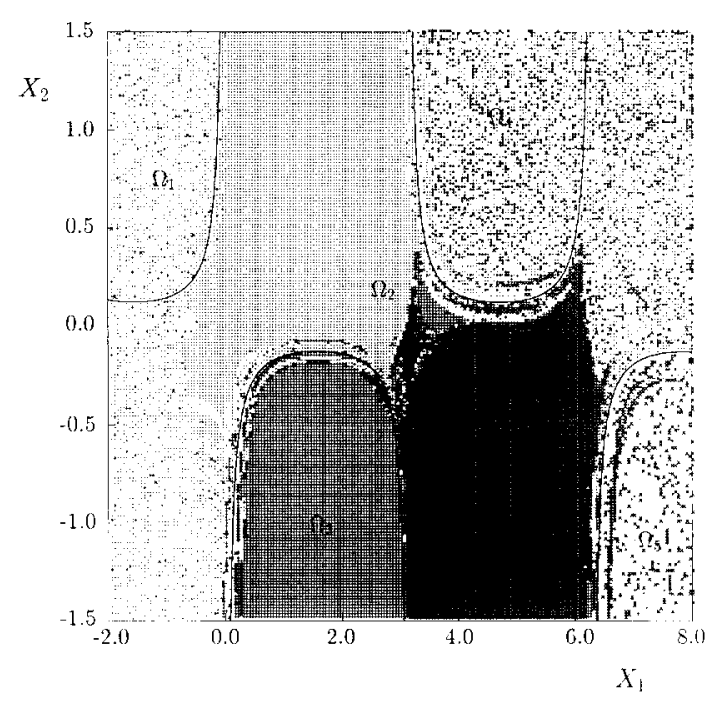

(b)

Fig. 16. (a) Location of equilibrium cells and fake periodic groups detected by the simple cell mapping method for Example 6. (b) Total domains of attraction of the stable equilibrium points for Example 6 obtained by the simple cell mapping method. Barrier manifolds are shown by solid lines. 


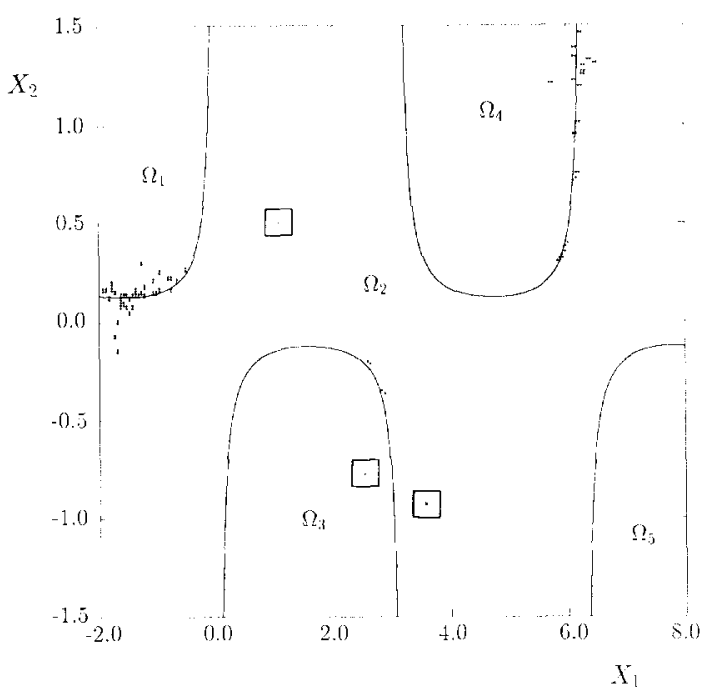

(a)

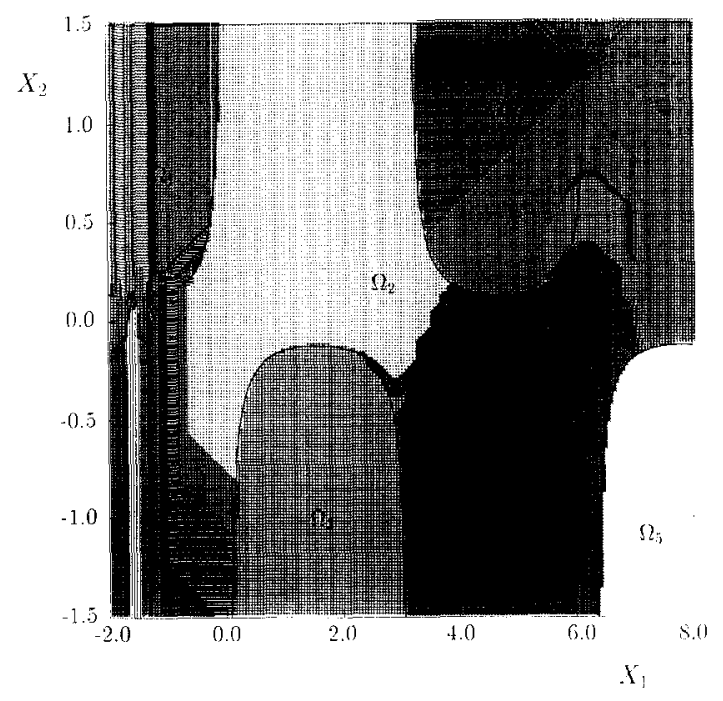

(b)

Fig. 17. (a) Location of equilibrium cells and fake periodic groups detected by the adjoining cell mapping method for Example 6. (b) Total domains of attraction of the stable equilibrium points for Example 6 obtained by the adjoining cell mapping method.

manifolds. The main difference between the two methods are in the domains of attraction of the equilibria of the system and the description of dynamics in the vicinity of the singular manifolds. Figure 17(b) shows the domains of attraction provided by the adjoining cell mapping method wherein the domain of attraction of each fake $P-2$ group is displayed by employing different symbols to indicate geometrically the manner in which many trajectories approach the barrier manifolds. The evolution of trajectories in the neighborhood of $\mathbf{x}_{S}^{(4)}$ (see the corresponding result in Figure 16(b) for the fake $P$-2 group indicated by symbol ' $*$ ') is clearly displayed by the domains of attraction associated with the corresponding fake $P-2$ groups. Also, the separation of the two domains of attraction in the isolated region $\Omega_{2}$ is remarkably clear in Figure 17(b). The domains of attraction of fake periodic groups indicate not only how trajectories flow very close to the barrier manifolds but they also show how far away trajectories map to them. Only a very small number of trajectories cross the barrier manifold which is again attributed to numerical inaccuracy during integration. Overall, the domains of attraction depicted by the adjoining mapping in Figure 17(b) method is considerably improved when compared with Figure 16(b) obtained by the simple cell mapping method.

To observe details close to one of the barrier manifolds, both the simple and adjoining cell mapping techniques are applied to the region $0.0 \leq x_{1}<6.0$ and $-5.0 \leq x_{2}<1.0$ containing $200 \times 200$ intervals with the same integration parameters as before. Figure 18 shows the domains of attraction of the three isolated equilibrium cells obtained by simple cell mapping analysis (no fake periodic groups are present in this case). We observe that there is a collection of cells around the singular manifold separating the regions $\Omega_{2}$ and $\Omega_{3}$. Even though it is quite difficult to glean from this figure, these cells appear to form a 'cone-like' region. Such a region is constituted by different disconnected portions of two (or more) domains of attraction. In the absence of fake periodic groups, the evolution of trajectories in the cone-region can be classified into three sets. 


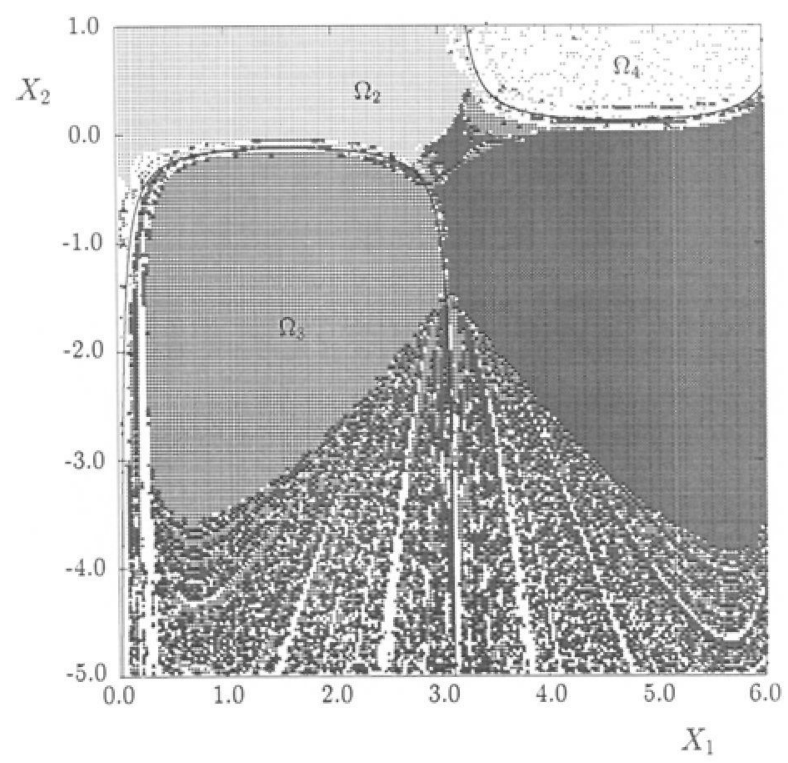

Fig. 18. An enlarged view of Figure 16(b) for Example 6 obtained by the simple cell mapping method.

One set apparently crosses the barrier manifolds due to numerical inaccuracy in integration (for example, computed trajectories evolving from cells marked with symbol ' + ' in region $\Omega_{2}$ eventually map to the equilibrium point $\mathbf{x}_{b}^{*}$ in $\Omega_{3}$ after crossing the barrier manifold; likewise, cells represented by symbol ' $x$ ' in region $\Omega_{3}$ map to the equilibrium point $\mathbf{x}_{c}^{*}$ in $\Omega_{2}$ ). The second set of trajectories approach the barrier manifold and, instead of crossing it, they are diverted back to map to the equilibrium point in the same region (for example, computed trajectories from cells marked ' + ' in region $\Omega_{3}$ approach the barrier manifold and eventually map to $x_{b}{ }^{*}$ located in the same region). Lastly, the third set consists of trajectories which behaves like those in the first and second sets and map to the sink cell as indicated by blank spaces in the cone-region. The totality of all these three sets of trajectories appear to form a 'cone-like' shape region in Figure 18 which will be made remarkably clear in Figure 19(b).

Figure 19(a) shows the location of equilibrium cells and fake periodic cells obtained by adjoining mapping analysis. Figure 19(b) displays the respective domains of attraction indicating that our method not only delineates the attraction domains associated with equilibria but they also reveal better the evolution of trajectories near the barrier manifold separating the regions $\Omega_{2}$ and $\Omega_{3}$. Referring to Figure 19(b), an interesting observation can be made of the domains of attraction associated with the groups of fake periodic cells found near the barrier manifold separating $\Omega_{2}$ and $\Omega_{3}$. They indicate that the trajectories evolving from the domain of attraction associated with each fake periodic group eventually hit the barrier manifold. Moreover, while doing so, these trajectories seem to evolve similarly for each group (that is, with similar slopes) and intersect the barrier manifold orthogonally (these facts have been verified by direct integration of the differential equation (1)). Once these trajectories hit the barrier manifold, their future dynamics would be undefined. The totality of the trajectories mapping to the fake periodic groups near this manifold appears to form a definitive shape ('cone-like' shape), the trajectories starting outside which region map eventually to other equilibrium points. To make this observation clear, only the domains of attraction corresponding to fake groups are shown in Figure 19(c) wherein the 


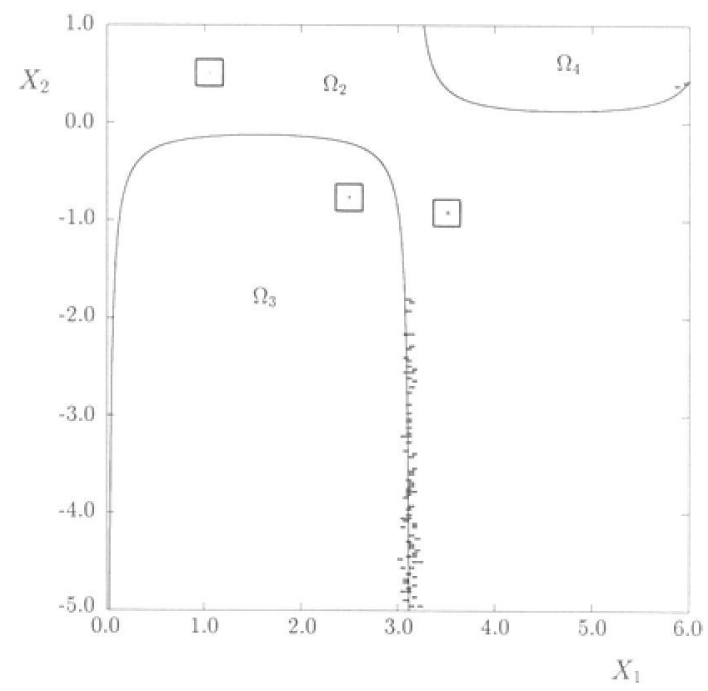

(a)

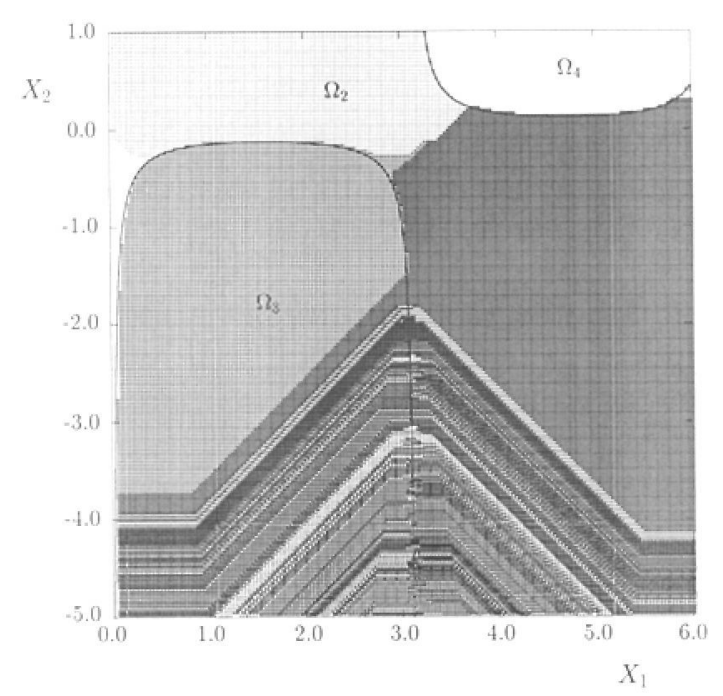

(b)

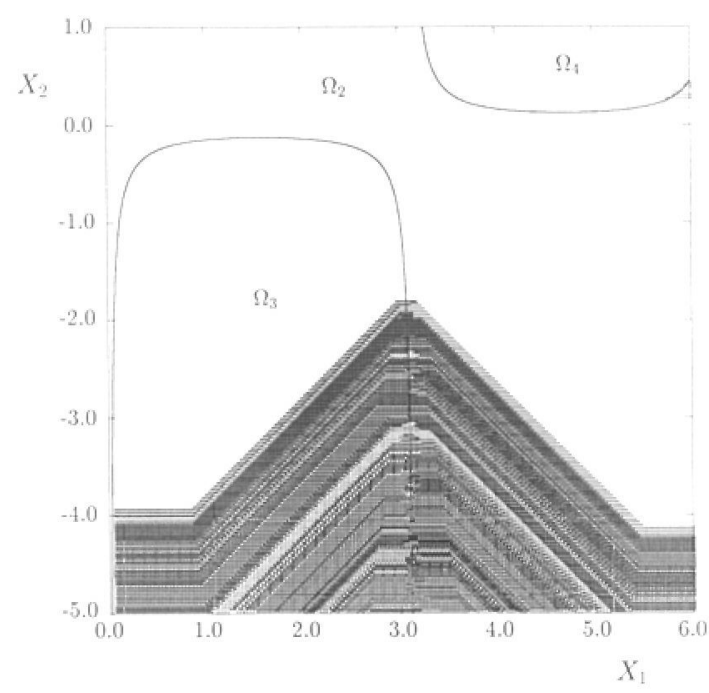

(c)

Fig. 19. (a) Location of equilibrium cells and fake periodic groups (indicated by symbol '*') detected by the adjoining cell mapping method for Example 6. (b) An enlarged view of Figure 17(b) for Example 6 obtained by the adjoining cell mapping method. (c) A portion of Figure 19(b) showing the domains of attraction of fake periodic groups near the barrier manifold separating the regions $\Omega_{2}$ and $\Omega_{3}$. The shape of the domain of attraction is 'cone-like' with the apex apparently on the barrier manifold displaying similarly evolving trajectories.

'cone-like' shape with its apex on the singular manifolds is obvious. We have also observed a similar shape of region near the barrier manifold separating $\Omega_{2}$ and $\Omega_{4}$. In addition to 'cone-like' regions, a 'center-like' region was also found on the singular manifold separating regions $\Omega_{1}$ and $\Omega_{2}$. We conjecture that such regions correspond to a class of extraneous singularities, for details see [12]. Thus, 'cone-like' shape together with 'center-like' shape of flow of trajectories near barrier manifolds are typical characteristics of the type of dynamical systems discussed here. 


\subsection{Example 7}

We consider the vector function cited in $[2,8]$ :

$$
\begin{aligned}
& f_{1}=x_{1}^{2}\left(x_{1}-1\right), \\
& f_{2}=x_{1}^{4}+x_{2}^{2}-a, \quad a \in \mathbf{R} .
\end{aligned}
$$

This example possesses a wide range of dynamic behavior depending on the value of the parameter $a$. By direct computation, we obtain the following vector field $\mathbf{F}$ for the dynamical system (1):

$$
\mathbf{F}(\mathbf{x})=\left[\begin{array}{c}
\frac{-x_{1}\left(x_{1}-1\right)}{3 x_{1}-2} \\
\frac{4\left(x_{1}-1\right) x_{1}^{4}-\left(3 x_{1}-2\right)\left(x_{1}^{4}+x_{2}^{2}-a\right)}{2\left(3 x_{1}-2\right) x_{2}}
\end{array}\right], \quad \operatorname{det} \mathbf{J}(\mathbf{x})=2\left(3 x_{1}-2\right) x_{1} x_{2}
$$

We only consider the case for which $a=1$. The three roots of $\mathbf{f}$ are $\mathbf{x}_{a}^{*}=(0,-1), \mathbf{x}_{b}^{*}=(0,1)$ and $\mathbf{x}_{c}^{*}=(1,0)$. There exist three singular manifolds given by $x_{1}=0, x_{1}=\frac{2}{3}$, and $x_{2}=0$. The roots $\mathbf{x}_{a}^{*}$ and $\mathbf{x}_{b}^{*}$ lie on the singular manifold $x_{1}=0$ where $\mathbf{F}$ is defined while the root $\mathbf{x}_{c}^{*}$ lies on the singular manifold $x_{2}=0$ where $\mathbf{F}$ is not defined. Hence, $\mathbf{x}_{a}^{*}$ and $\mathbf{x}_{b}^{*}$ are equilibria of (1) while $\mathbf{x}_{c}^{*}$ is not an equilibrium point of (1).

The state space region under study for the simple cell mapping method is $-2.0 \leq x_{1}<2.0$ and $-2.0 \leq x_{1}<2.0$ which is divided into $300 \times 300$ intervals. Integration parameters are $T_{S}=1$ unit and $\Delta t=T_{S} / 15$. The computational results indicate that there are four adjoining $P-1$ cells representing each of the equilibrium points $\mathbf{x}_{a}^{*}$ (symbol " + ") and $\mathbf{x}_{b}^{*}$ (symbol " $x$ ") while the point $\mathbf{x}_{c}^{*}$ (symbol ".") is replaced by one group of adjoining $P-2$ cells. The total domains of attraction obtained by the simple cell mapping for the periodic cells are shown in Figure 20. Cell represented

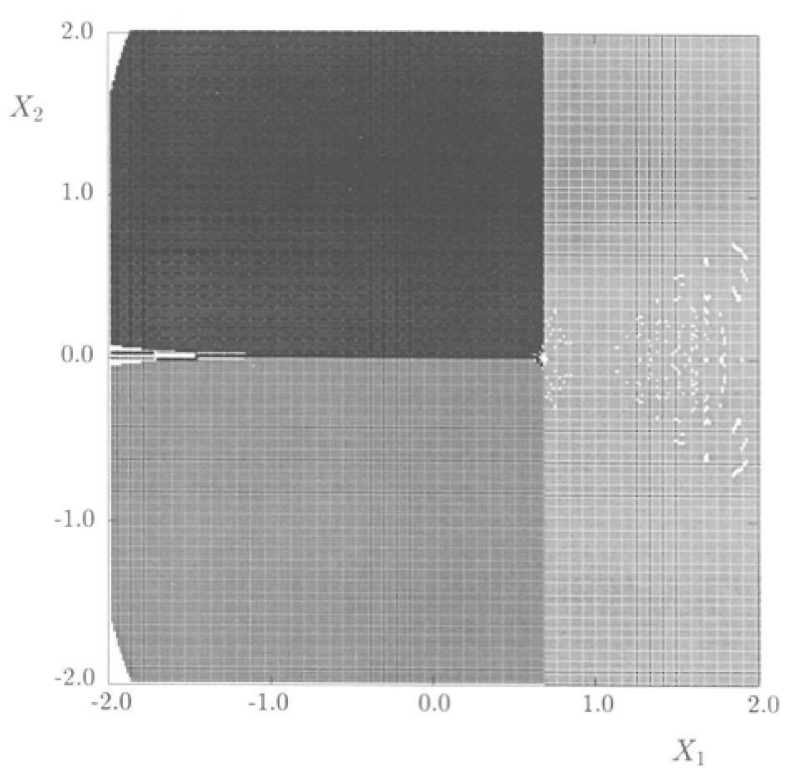

Fig. 20. Total domains of attraction of the stable equilibrium points and pathological points for Example 7 obtained by the simple cell mapping method. 
by a blank is mapped to the sink cell. Note the disconnected domain of attraction corresponding to the point $\mathbf{x}_{c}^{*}$. As before, some cells cross the manifold $x_{2}=0$. There is also a set of cells mapping to the sink cell in the neighborhood of the point $\mathbf{x}_{c}^{*}$ forming a "cone-like" shape. The simple cell mapping method is unable to clarify the domains of attraction close to the manifold $x_{2}=0$.

The results of adjoining cell mapping method for this example are shown in Figures 21(a)-(b) where we have used the same cell mapping parameters as above with initial integration parameters $T=1$ and $\Delta t=T / 15$. Figure 21(a) shows the location of equilibrium cells representing $\mathbf{x}_{a}^{*}$ (symbol ' + '), $\mathbf{x}_{b}^{*}$ (symbol ' $x^{\prime}$ ) and $\mathbf{x}_{c}^{*}$ (symbol ' ') which are same as before. In addition, there are 51 groups of fake P-2 cells (symbol '*'). Figure 21(b) shows the domains of attraction for the periodic cells. The collection of trajectories in the neighborhood of the point $\mathbf{x}_{c}^{*}$ (which is on the singular manifold) and mapping to it form a 'funnel-like' region which is symmetric with respect to the $x_{1}$-axis (this region is identifiable in Figure 21(b) within the enclosed box). This 'cone-like' shape of region of attraction was previously predicted analytically by performing a local analysis in the neighborhood of $\mathbf{x}_{c}^{*}$, see Zufiria [8]. It appears that the extent of the predicted 'cone-like' domain of attraction exceeds well beyond that expected from a linear analysis. When compared with the results of the simple cell mapping method (Figure 20), the new technique introduced here very clearly displays the various domains of attraction. Also, observe the clarity of the regions of attraction near the singular manifold $x_{2}=0$.

In summary, the technique of adjoining cell mapping provides a 'cleaner' and computationally expected description of dynamics near singular manifolds for nonstandard dynamical systems studied in this section. Overall, it better portrays the analytical properties possessed by such systems, specifically the dynamics of trajectories which hit singular manifolds. While the simple cell mapping method is unable to clarify the dynamics of such trajectories, the adjoining mapping method is capable of complementing analytical findings.

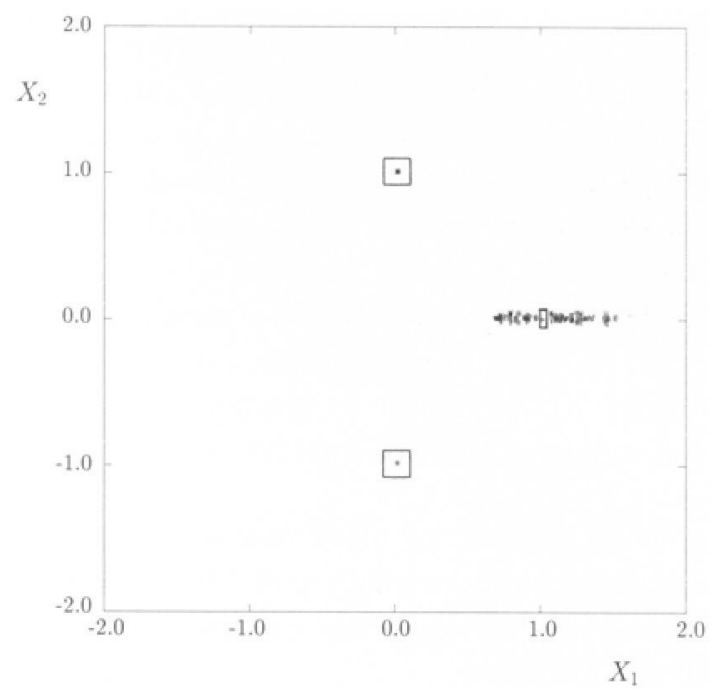

(a)

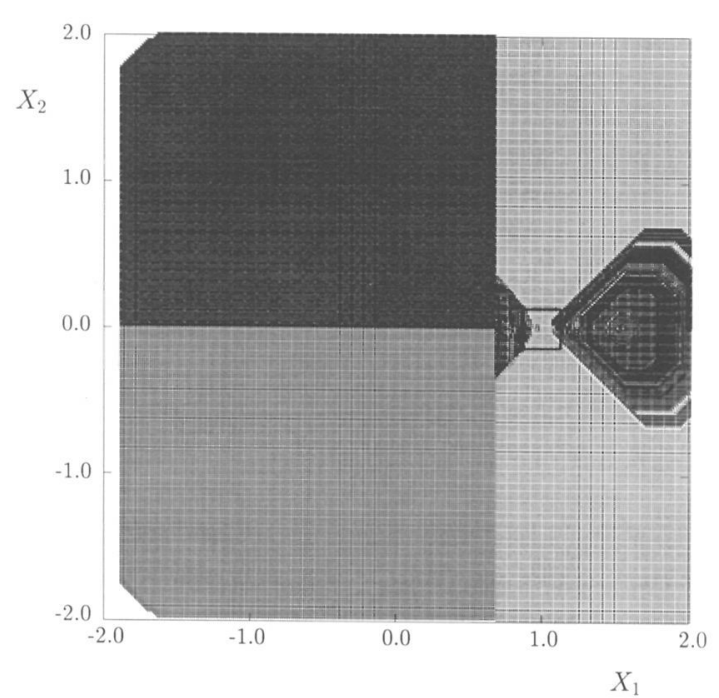

(b)

Fig. 21. (a) Location of equilibrium cells and fake periodic groups (indicated by symbol '*') detected by the adjoining cell mapping method for Example 7. (b) Total domains of attraction of the stable equilibrium points and pathological points for Example 7 obtained by the adjoining cell mapping method. The boxed region indicates 'cone-like' attraction domain for the point $\mathbf{x}_{c}^{*}=(1,0)$ which is a zero of $\mathbf{f}$ and lies on the singular manifold $x_{2}=0$. 


\section{Comparative Properties}

The new procedure of adjoining cell mapping proposed by Zufiria and Guttalu [11] and applied in this paper has clearly shown its capability for analyzing global behavior of both smooth and nonstandard autonomous dynamical systems. This technique has several attractive features and advantages when compared with the simple cell mapping method as described below.

\subsection{Features of Adjoining Cell Mapping}

1. The influence of the duration of integration $T$ is minor in the final results.

2. In general, it is difficult to compare directly the computational performance of the simple cell mapping and adjoining mapping methods as the latter utilizes a variable integration duration which differs from cell to cell. Since more than one iteration is required for estimating the duration of integration $T$ for each cell, it is obvious that more computational time is needed to determine an adjoining mapping than its counterpart the simple cell mapping (provided that the same fixed number of integration steps is employed for both methods). For the set of integration parameters used in the examples studied here, the adjoining mapping method was significantly faster than the simple cell mapping method because the latter used a large number of integration steps. One fair comparison we have made is to employ one-step Euler integration scheme for both methods in which case $\mathbf{F}(\mathbf{x})$ is evaluated just once for each step of integration. For the examples presented in this paper, we found out that the adaptive mapping unraveling algorithm required from 0.9 to 1.5 times the amount of computational time than that required by the simple cell mapping unraveling algorithm. Whereas the adjoining technique provided meaningful results with one-step Euler integrator, the simple cell mapping method often missed limit cycles and its results were difficult to interpret.

The initial estimate of the adjoining mapping time defined in [11] affects the amount of computation required. Such a time estimate can be taken from the previously computed cell-time (or better, from an adjoining cell if its cell-time has already been computed and stored in memory). This additional adaptive scheme can provide a substantial reduction in computation time. In practical implementation, an adaptive selection of the first time estimate makes this procedure only about a few times slower than the simple cell mapping method when a fourth-order Runge-Kutta integration is employed. On the other hand, a robust adaptation of the simplified scheme proposed in Section 3.4 of [11] speeds up the performance of the adjoining cell mapping method by more than an order of magnitude and makes it faster than the simple cell mapping method using a fourth-order Runge-Kutta integration. This new scheme is also sometimes faster than the simple cell mapping method employing a simplified integration scheme.

3. When detecting limit cycles, a simple cell mapping may replace it by more than one group of periodic cells and the number of groups and the location of periodic cells are highly dependent on the integration time $T_{S}$. An adjoining mapping usually provides only one periodic orbit associated with a limit cycle as demonstrated in all the examples.

4. The discretization error for an adjoining cell mapping may be more pronounced than that for a simple cell mapping, especially when asymptotically stable invariant sets are present. On the other hand, when unstable invariant sets exist, discretization error due to a simple cell mapping may be accumulated rapidly during a long time integration making it unable to detect them. This is not the case for an adjoining cell mapping which locates unstable invariant sets in many cases because it is defined by considering only an adjoining time of integration. 
5. When studying systems of the form analyzed in $[2,8,9,10]$, the simple cell mapping method has tremendous difficulties especially close to the singular manifolds. Adjoining cell mapping overcomes these difficulties by always preserving an adjoining mapping among all the cells. Hence, this new technique is able to provide invaluable information regarding the evolution of trajectories in the critical regions. It also delineates better the domains of attraction since the connectedness property of every domain of attraction of the original system is preserved. Finally, it has been instrumental in providing the crucial information like cone-like domains of attraction of some roots of $\mathbf{f}$ located on singular manifolds (as predicted by linear analysis near the manifold).

\subsection{Features of Recursive Unraveling Algorithm}

1. As illustrated in Example 1, the recursive unraveling algorithm is shown to reduce the memory requirement drastically for computing invariants of the system when compared with the simple cell mapping unraveling algorithm provided in [3].

2. A recursive version of the unraveling algorithm for adjoining cell mapping has been implemented using the $C$ programming language and found to be very compact.

3. A proper choice of the partition (especially at the first level) can make the boundaries of the blocks to be defined along state space variables requiring less number of cells. Therefore, a reduction in memory by a factor of $n_{\max } / 2$ can be accomplished (where $n_{\max }$ is the maximum number of interval-divisions required along a state space variable).

\section{Concluding Remarks}

The adjoining cell mapping method developed in [11] has been applied to different classes of examples. The ability of the new technique to locate limit cycles is clearly established. Not only can the method locate limit cycles very effectively, but also its computational results are very easy to interpret in the case of dynamical systems possessing limit cycles. When applied to Hamiltonian systems, the appearance of numerous contiguous periodic groups of cells which are uncommon in adjoining mapping results suggest the presence of dynamics peculiar to conservative systems. On the other hand, the detection of several interlaced periodic cells for a chaotic system which are again uncommon to adjoining mapping results may suggest the presence of interesting dynamics requiring further study. The reduction of memory requirements due to the recursive nature of the unraveling algorithm has also been illustrated. The strength of the adjoining cell mapping technique in analyzing global dynamics of special class of dynamical systems has also been indicated.

Further research in the development and efficient implementations of adaptive and recursive unraveling of the adjoining cell mapping will provide a needed computational tool for the analysis of nonlinear mechanical systems of at least three degrees of freedom. Also, its application to generalized cell mapping method (see [4]) will provide another effective tool for analyzing probabilistic dynamics.

\section{Acknowledgements}

The work reported here is partially supported by a grant from the National Science Foundation and the USC Faculty Research and Innovation Fund. P. J. Zufiria also thanks the support of a 
Formación de Postgrado fellowship of the Programa Nacional de F.P.I. provided by the D.G.I.C.T. of the Ministerio de Educación y Ciencia of Spain.

\section{Note}

1. For a detailed description of the barrier manifolds and their influence on the global behavior of the type of dynamical systems examined in this section, the reader is referred to [9]. It suffices to mention here that a barrier manifold is a singular manifold with the additional property that the system trajectories cannot cross it.

\section{References}

1. Dewey, A. G. and E. I. Jury, 'A note on Aizerman's conjecture', IEEE Transactions on Automatic Controls AC-10, 1965, 481-482.

2. Guttalu, R. S. and P. J. Zufiria, 'On a class of nonstandard dynamical systems: Singularity issues', in C. T. Leondes (ed.), Control and Dynamic Systems 34, 1990, 279-324.

3. Hsu, C. S. and R. S. Guttalu, 'An unravelling algorithm for global analysis of dynamical systems: An application of cell-to-cell mappings', Journal of Applied Mechanics 47, 1980, 940-948.

4. Hsu, C. S., Cell-to-Cell Mapping: A Method of Global Analysis for Nonlinear Systems, Springer-Verlag, New York, 1987.

5. Lorenz, E. N., 'Deterministic nonperiodic flow', Journal of Atmospheric Sciences 20, 1963, 130-141.

6. Reinhall, P. G., T. K. Caughey, and D. W. Storti, 'Order and chaos in a discrete Duffing oscillator: implications on numerical integration', ASME Journal of Applied Mechanics 56, 1989, 162-167.

7. Sparrow C. T., The Lorenz Equations: Bifurcations, Chaos, and Strange Attractors, Springer-Verlag, New York, 1981.

8. Zufiria P. J., 'Global behavior of a class of nonlinear dynamical systems: Analytical, computational and control aspects', Ph.D. Dissertation, University of Southern California, 1989.

9. Zufiria, P. J. and R. S. Guttalu, 'On an application of dynamical system theory to determine all the zeros of a vector function', Journal of Mathematical Analysis and Applications 152, 1990, 269-295.

10. Zufiria, P. J. and R. S. Guttalu, 'A computational method for locating all the roots of a vector function', Applied Mathematics and Computation 35, 1990, 13-59.

11. Zufiria, P. J. and R. S. Guttalu, 'The adjoining cell mapping and its recursive unraveling, Part I: Description of adaptive and recursive algorithms', Nonlinear Dynamics 4, 1993, 207-226.

12. Zufiria, P. J. and R. S. Guttalu, 'The role of singularities in Branin's method', Dynamics and Control (submitted for publication). 